\title{
On ultrasound-induced microbubble oscillation in a capillary blood vessel and its implications for the blood-brain barrier
}

\author{
W Wiedemair ${ }^{1}$, Ž Tuković ${ }^{2}$, H Jasak Th $^{2,3}$, D Poulikakos ${ }^{1}$ \\ and V Kurtcuoglu ${ }^{1}$ \\ ${ }^{1}$ Laboratory of Thermodynamics in Emerging Technologies, Department of Mechanical and \\ Process Engineering, ETH Zurich, Zurich, Switzerland \\ ${ }^{2}$ Faculty of Mechanical Engineering and Naval Architecture, University of Zagreb, Zagreb, \\ Croatia \\ ${ }^{3}$ WIKKI Ltd, London, UK \\ E-mail: vartan.kurtcuoglu@ethz.ch
}

Received 29 July 2011, in final form 5 December 2011

Published 1 February 2012

Online at stacks.iop.org/PMB/57/1019

\begin{abstract}
The complex interaction between an ultrasound-driven microbubble and an enclosing capillary microvessel is investigated by means of a coupled, multidomain numerical model using the finite volume formulation. This system is of interest in the study of transient blood-brain barrier disruption (BBBD) for drug delivery applications. The compliant vessel structure is incorporated explicitly as a distinct domain described by a dedicated physical model. Red blood cells (RBCs) are taken into account as elastic solids in the blood plasma. We report the temporal and spatial development of transmural pressure $\left(P_{\mathrm{tm}}\right)$ and wall shear stress (WSS) at the luminal endothelial interface, both of which are candidates for the yet unknown mediator of BBBD. The explicit introduction of RBCs shapes the $P_{\mathrm{tm}}$ and WSS distributions and their derivatives markedly. While the peak values of these mechanical wall parameters are not affected considerably by the presence of RBCs, a pronounced increase in their spatial gradients is observed compared to a configuration with blood plasma alone. The novelty of our work lies in the explicit treatment of the vessel wall, and in the modelling of blood as a composite fluid, which we show to be relevant for the mechanical processes at the endothelium.
\end{abstract}

S Online supplementary data available from stacks.iop.org/PMB/57/1019/mmedia

(Some figures may appear in colour only in the online journal)

\section{Introduction}

The blood-brain barrier (BBB) is a functional structure at the interface between blood and neural tissue to protect the brain from exposure to potentially toxic substances and to maintain 
the homeostasis of its microenvironment. It regulates species exchange and inhibits the transfer of most substances from blood to the central nervous system (CNS) (Abbott et al 2010). This effect arises mainly from the presence of tight junctions (TJs) that seal the intercellular cleft between endothelial cells of the CNS vasculature (Dejana 2004), forming a physical barrier that severely limits paracellular transport. The BBB inhibits the transfer of virtually all large molecules and the vast majority of small molecules (Dove 2008) depending on their lipid solubility and charge (Fenart et al 1999). While this functionality is essential for keeping pathogens and toxic substances away from the brain, it also impedes the passage of most systemically administered therapeutic substances. As a result, medications for several cerebral ailments cannot be readily delivered (Abbott and Romero 1996).

Microbubble (MB) enhanced focused ultrasound (FUS) application in cerebral capillary blood vessels is crystallizing as a promising approach for localized, transient opening of the BBB (Vykhodtseva et al 2008, Hynynen 2008). This method employs pre-formed gas MBs located inside a microvessel which respond to an incident ultrasound field by undergoing forced volumetric oscillations that alter the local pressure and shear conditions. A successful application of this approach would allow for the targeted delivery of any drug as well as other substances such as nanoparticles (Liu et al 2010, Lockman et al 2002) and contrast agents (Howles et al 2010) to the CNS with minor disturbance of the BBB's protective function. The transient character of this method and its reversible effects on tissue and functionality were shown in several studies (Alonso et al 2010, Howles et al 2010). A successful application of the MB enhanced FUS treatment in small animals (Hynynen et al 2001, Choi et al 2007) as well as more recently in non-human primates (Marquet et al 2011) has been reported. While various ways by which this process referred to as BBB disruption (BBBD) may occur have been suggested, the exact mechanisms are yet to be fully elucidated (Meairs and Alonso 2007).

Mechanical stress and strain on the endothelium caused by forced MB oscillation is one of the main purported BBBD mechanisms. Pathways for transport across the BBB may open in the form of fenestrae in the endothelium, cytoplasmic channels, widening of the intercellular clefts with disruption of TJs or in the form of damaged endothelial areas (Sheikov et al 2004, Meairs and Alonso 2007). Furthermore, an increase in the number of vesicles in sonicated regions may augment the rate of transcytosis (Sheikov et al 2004, Hynynen 2008). Shear stresses created by bubble microstreaming may trigger a biological response from nearby endothelial cells (Nyborg 2001), eventually contributing to BBBD (Collis et al 2010, Vykhodtseva et al 2008). All these potential effects can be attributed fully or in part to the occurrence of mechanical forces at the endothelium, thus warranting investigation of the mechanical conditions in that region. The isolation and quantification of the involved forces may allow for an identification of cause-and-effect chains with respect to the suggested BBBD mechanisms. This study aims at contributing to this process through the numerical investigation of a single ultrasound-driven MB in a capillary vessel.

MBs have been used for decades as contrast agents for diagnostic ultrasound (Stride and Saffari 2003), acting as scattering centres by virtue of the large difference in density and compressibility between MB and surrounding liquid. Generally, the same MBs are utilized in FUS-driven BBBD (Kaul 2008, Tu et al 2009, Sponheim et al 1993). Their small diameter of 2-8 $\mu \mathrm{m}$ enables the MBs to pass through narrow capillaries. A shell encapsulation of their lowdiffusivity gas filling adds to their stability and prolongs circulation time after application. The composition and thickness of the shell, as well as the nominal MB radius, determine acoustic and echogenic properties. The resonance frequency, for example, can be tuned within the $\mathrm{MHz}$ range (Stride 2005). 
The comportment of MBs when exposed to an ultrasound field has been investigated extensively both theoretically and experimentally for unconstrained (Plesset and Prosperetti 1977) and encapsulated MBs (Church 1995, Frinking and de Jong 1998, Marmottant et al 2005). Direct observation employing ultra high speed cameras was also achieved (de Jong et al 2000, Chin et al 2003). The two general cases of stable volumetric oscillation and inertial cavitation (IC) of the MB have to be distinguished. IC is often associated with severe biological damage such as vessel rupture and extravasation induced through jet formation, shockwaves and high local temperatures generated during the MB collapse (Miller et al 1996, Baseri et al 2010, McDannold et al 2006). IC can be detected in vivo by analysing the broadband response in the acoustic emissions of the ultrasound contrast agent (UCA) (Tu et al 2006, Tung et al 2010).

For medical applications, MBs experience a confined geometry inside small blood vessels, which impacts their dynamics significantly. The case of unconstrained gas bubbles in rigid tubes has been studied theoretically (Sassaroli and Hynynen 2004) and treated by means of a modified Rayleigh-Plesset equation (RPE) (Zudin 1992, Klotz and Hynynen 2010). Taking into account vessel compliance, a lumped parameter model has been employed to investigate MB interaction with the vessel wall (Qin and Ferrara 2006, 2007). The effect of MB oscillation in vessels has also been studied experimentally in gel phantoms (Caskey et al 2009), including direct observations with high speed cameras (Zheng et al 2007). An excellent review of the topic is found in Qin et al (2009).

Next to MB parameters, vessel confinement and liquid properties, the acoustic parameters of the incident ultrasound field, such as frequency $(f)$ and peak negative pressure (PNP), i.e. the peak rarefaction pressure during the negative half-cycle of the ultrasound field, define the response of the MB (McDannold et al 2008b). The mechanical index (MI) $=\mathrm{PNP}(\mathrm{MPa}) / f$ $(\mathrm{MHz})^{1 / 2}$ was introduced as a measure for IC threshold (Apfel and Holland 1991) and is also well suited to characterize BBB opening (McDannold et al 2008a). Reported values of the threshold MI for BBBD range from 0.25 (Baseri et al 2010, Tung et al 2010) to 0.46 (McDannold et al 2008a), while the threshold for IC ranges between 0.4 (Tung et al 2010) and 0.78 (McDannold et al 2006). Consequently, IC can be involved in BBBD, but does not appear to be a prerequisite. Our model applies exclusively to the stable cavitation of MBs.

The oscillation of MBs in blood vessels has to be well controlled by means of the ultrasound parameters in order to avoid permanently damaging the blood vessel and BBB through extravasation or haemorrhage (McDannold et al 2005, Hynynen et al 2001). The assessment of endothelial mechanical conditions for different ultrasound configurations and MB properties may help to quantify the impact of the choice of those parameters.

In view of the expected close correlation between mechanical stresses acting on the endothelium and an opening of suspected BBBD pathways, a microscale modelling approach with focus on the luminal vessel interface is proposed. The novelty of this work lies in the explicit incorporation of the vessel structural dynamics and its interaction with the luminal liquid into a numerical scheme for a coupled multi-domain system. This yields a physically comprehensive model that grants access to relevant mechanical parameters such as transmural pressure $\left(P_{\mathrm{tm}}\right)$ and wall shear stress (WSS) based on the properties of the vessel, the liquid and the MB. Moreover, the impact of the presence of red blood cells (RBCs) in the vicinity of the MB on these mechanical conditions is addressed for the first time. Our work aims to predict the transient mechanical state of a microvessel during MB enhanced FUS application. Its rationale is that knowledge of the mechanical state at the luminal endothelial interface will be of advantage for determining an ultrasound parameter space within which safe BBB disruption can take place. 
compliant capillary $\left(\rho \mathrm{E} v_{\rho}\right)$

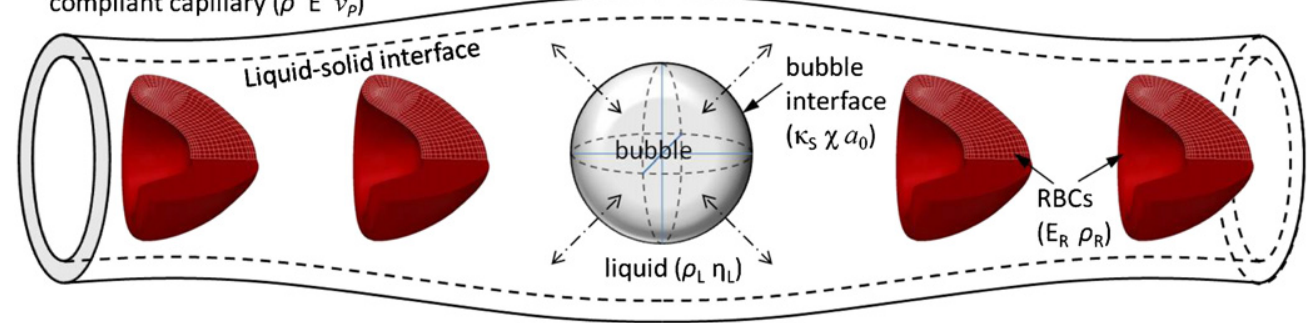

Figure 1. Illustration of the geometric setup. A single MB, enclosed by RBCs on both sides, is located at the centre of a compliant, cylindrical tube section representing a capillary. The MB is characterized by its equilibrium radius $a_{0}$, shell dilatational viscosity $\kappa_{S}$ and elastic shell compression modulus $\chi$. The RBCs are modelled using density $\rho_{R}$ and Young's modulus $E_{R}$. The liquid is described as Newtonian and incompressible with density $\rho_{L}$ and dynamic viscosity $\eta_{L}$. The compliant tube is modelled as an elastic solid and defined by its density $\rho$, Young's modulus $E$ and the Poisson ratio $v_{P}$. A liquid-solid interface is present at the luminal endothelium surface and the RBC surface.

\section{Materials and methods}

The microscopic system investigated in this study consists of a section of a capillary vessel filled with blood plasma and a single MB at its centre surrounded by RBCs at either axial side of the MB (figure 1). The MB couples to an incident ultrasound field and is forced into volumetric oscillation. The thereby induced transient displacement of blood plasma interacting with the compliant vessel wall and the adjacent RBCs results in microcirculations that create local pressure variations, shear forces on the endothelium and vessel dilation.

The three coupled domains, namely MB, blood plasma as well as vessel wall and RBCs, are described by distinct physical models that interact as illustrated in figure 3. Each domain is treated using a dedicated numerical solver within OpenFOAM version 1.6-ext (Weller et al 1998, Jasak et al 2007), an open source C++ class library for continuum mechanics simulations.

\subsection{General assumptions}

We assume bulk blood flow through the capillary vessel to be decoupled from the local microcirculation caused by the MB oscillation: blood propagates through capillary vessels at speeds of the order of $1 \mathrm{~mm} \mathrm{~s}^{-1}$ (Fischer et al 1996, Mathura et al 2001, Stucker et al 2004), while the MB surface velocity reaches values of the order of $1 \mathrm{~m} \mathrm{~s}^{-1}$ at ultrasound excitation frequencies in the $\mathrm{MHz}$ range. $\mathrm{MB}$ advection with bulk blood flow is thus insignificant within the time frame of a few MB oscillation cycles (Miao et al 2008).

We further assume a spatially uniform ultrasound pressure field: the ultrasound wavelength is of the order of $1 \mathrm{~mm}$ and thus much larger than the dimension of the region of interest surrounding the MB (Qin and Ferrara 2006).

The MB is positioned at the centre of the tube and retains a spherical shape throughout the simulation. Neglecting the asphericity due to geometric confinement is justified by the use of small MBs and low expansion ratios (Stride and Saffari 2003). This is particularly applicable when taking into account the stabilizing effect of the viscoelastic encapsulation that increases the MB stiffness significantly (Hoff et al 2000). Moreover, the focus of this study is on the mechanical state of the endothelium, which is determined by the far-field flow induced by the MB oscillation. This flow is governed by the overall volume displacement rate rather than by 


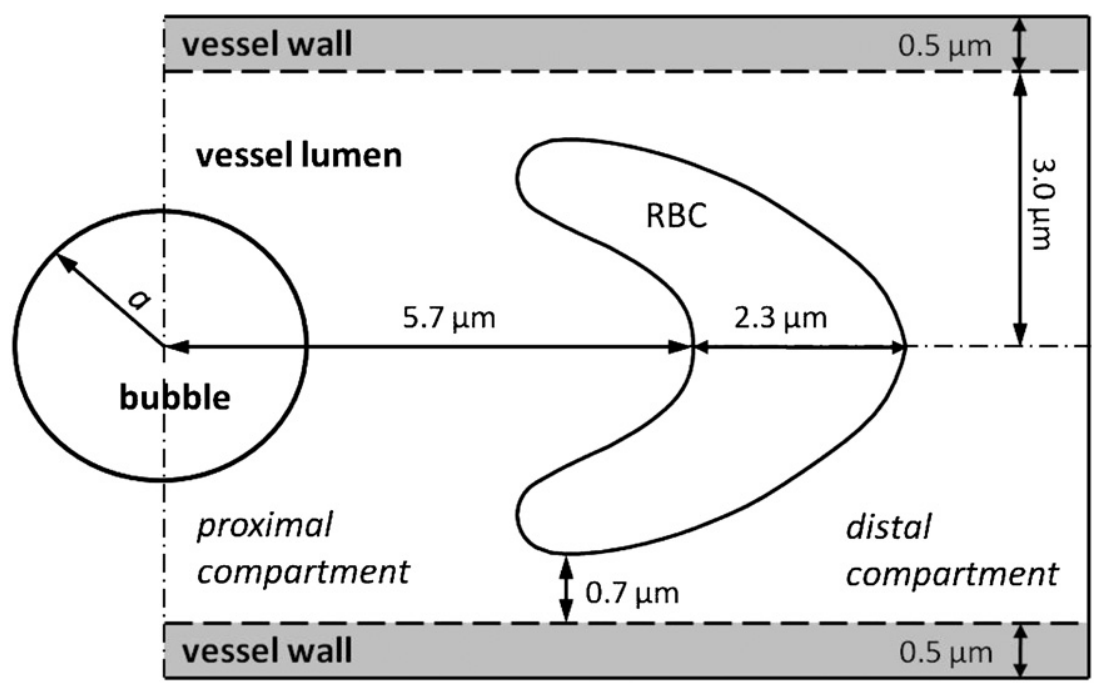

Figure 2. Configuration of the computational domain. Key dimensions are indicated. $a$ denotes MB radius.

the exact MB shape. Given a perfectly axisymmetric setup and not taking translation due to radiation pressure into account, the MB will keep its central position within the tube.

\subsection{Computational domain}

A right circular cylindrical tube of $20 \mu \mathrm{m}$ length with a lumen diameter of $6 \mu \mathrm{m}$ (Meier-Ruge et al 1980, Bertossi et al 1997, Cassot et al 2006) and wall thickness of $0.5 \mu \mathrm{m}$ (Bertossi et al 1997, Hogan et al 1986) represents the structural part of a capillary vessel section. It encloses the liquid-filled vessel lumen containing a spherical void at its centre, which represents the MB (figure 2). RBCs are positioned on both sides of the MB with a pitch of $5.7 \mu \mathrm{m}$ partially obstructing the vessel. The pitch is derived from considerations about the mixing of RBCs and MBs, as well as species concentrations as detailed in section 2.6. The RBCs adopt a coaxial parachute-like shape (Noguchi and Gompper 2005) to squeeze through the narrow capillary tube. This divides the computational domain in a proximal vessel compartment in the vicinity of the MB and a distal compartment.

The three-dimensional computational domain can be reduced to a narrow wedge by introducing boundary conditions accounting for the cylindrical symmetry of the system. Planar symmetry with respect to the central cross-section of the vessel is assumed. While geometrically not exact, the significant precedence of the flow induced by the MB over a possible cross-flow between upstream and downstream side justifies this measure. Consequently, it is sufficient to solve the governing equations on a wedge domain of half of the length of the original tube section.

We use a standard cylindrical coordinate system to specify positions within the geometry, where $r$ represents radial, $\Theta$ azimuthal and $z$ longitudinal axial location. The coordinate origin is placed in the centre of the MB.

The solid domain (vessel wall and RBC) and the liquid domain (vessel lumen) are resolved using two distinct unstructured computational grids composed entirely of hexahedral cells or control volumes. The gas domain (MB) has a well-defined spatial configuration, within which uniform pressure is assumed. This renders the use of a mesh for spatial discretization obsolete. 
Temporal discretization is performed with uniform time steps of $\Delta t=2 \mathrm{~ns}$ (see supplemental material available at stacks.iop.org/PMB/57/1019/mmedia for details).

The displacement of the gas-liquid interface as well as the motion of the solid-liquid interface require an adaptation of the computational grids to retain optimal mesh quality. This is performed through dynamic mesh adaptation using Laplace point diffusion in conjunction with tetrahedral cell decomposition, as well as topological mesh modification at run-time. The internal vertices are moved based on the prescribed motion of the boundary vertices using a vertex-based automatic mesh motion solver (Jasak and Tuković 2007, Tuković 2005). In order to preserve the temporal accuracy on the moving grid, mesh motion fluxes are calculated in a way that satisfies the space conservation law (Farhat and Geuzaine 2004, Geuzaine et al 2003).

\subsection{Ultrasound field}

The waveform of the incident ultrasound field is prescribed and assumed to be sinusoidal. Other patterns would be equally well implementable. The sonication frequency was set to $0.55 \mathrm{MHz}$ with PNP of $300 \mathrm{kPa}$, which corresponds to a MI of 0.4. The chosen example values together with the respective setup parameters yield stable MB cavitation when introduced into the RPE (see section 2.4) and are in the range of ultrasound parameters generally applied for BBBD (McDannold et al 2006, Zheng et al 2007, Hynynen et al 2005).

\subsection{Microbubble model}

An UCA MB consists of a gas core usually encapsulated by a shell made of human albumin, lipids or polymers (Kaul 2008). The filling gas is typically largely insoluble in blood and can be described by a polytropic law (Prosperetti 1991). Fluorocarbons are often used for this purpose. For most types of UCAs such as Definity ${ }^{\mathrm{TM}}$ (Lantheus Medical Imaging, North Billerica, MA, USA), SonoVue ${ }^{\mathrm{TM}}$ (Bracco, Milan, Italy) or Sonazoid ${ }^{\mathrm{TM}}$ (GE Healthcare, Chalfont St Giles, UK), the MB equilibrium diameter is distributed between 1 and $10 \mu \mathrm{m}$, with a mean of approximately 2-3 $\mu \mathrm{m}$ around which the diameter of the vast majority of the population is located (Faez et al 2011, Greis 2004, Sontum 2008, Goertz et al 2007).

In our model, the MB is regarded as an actuator that drives the surrounding liquid. The coupling to the incident ultrasound field and the impact of the surrounding liquid is treated implicitly through a modified RPE (Plesset and Prosperetti 1977). The original RPE (1) describes the dynamics of a single, spherical bubble in an infinite fluid:

$$
\rho_{L}\left(a \ddot{a}+\frac{3}{2} \dot{a}^{2}\right)=P_{g}-P_{\infty}-\frac{2 \sigma}{a}-\frac{4 \eta_{L} \dot{a}}{a} .
$$

Here, $a$ is the radius of the spherical bubble as a function of time. Dots and double dots over variables indicate, respectively, first and second derivative with respect to time. $\rho_{L}$ and $\eta_{L}$ designate liquid density and dynamic viscosity, respectively, and $\sigma$ is the surface tension. $P_{g}$ and $P_{\infty}$ are the internal gas pressure and the liquid pressure at infinity, respectively. The right-hand side of equation (1) represents $\Delta P$, which is the difference between the pressure at the MB surface and $P_{\infty}$.

An unconstrained bubble with uniform internal pressure obeying a polytropic gas law in an incompressible fluid and exposed to an arbitrary, time-dependent acoustic pressure may be described by substituting the following right-hand side into equation (1):

$$
\Delta P=\left(P_{0}-P_{v}+\frac{2 \sigma}{a_{0}}\right)\left(\frac{a_{0}}{a}\right)^{3 \kappa}-\frac{2 \sigma}{a}-\frac{4 \eta_{L} \dot{a}}{a}-P_{0}+P_{v}-P_{\mathrm{US}}(t) .
$$


Here, $a_{0}$ stands for the equilibrium bubble radius, $P_{0}, P_{v}$ and $P_{\mathrm{US}}$ are the ambient pressure, the vapour pressure and the incident ultrasound pressure, respectively, and $\kappa$ is the polytropic exponent. Assumed values for the polytropic exponent of the filling gas range from isothermal condition at $\kappa=1.0$ (Stride and Saffari 2004) to adiabatic at $\kappa=1.4$ (Qin and Ferrara 2006). We used a value of $\kappa=1.0$.

According to the work of Zudin (1992) and Klotz and Hynynen (2010), the left-hand side of equation (1) can be modified to account for the influence of the confining vessel by introducing an effective mass, resulting in equation (3). To include the dynamics of a bubble shell, the right-hand side of equation (2) is further adapted according to Hoff et al (2000), yielding equation (4). This model describes the dynamics of a gas-filled bubble with a very thin viscoelastic shell in an incompressible, Newtonian fluid. The shell properties are assumed constant throughout the oscillation.

$$
\begin{aligned}
& \rho_{L} a \ddot{a}\left(1+\frac{a L}{R_{T}^{2}}\right)+\frac{3}{2} \rho_{L} \dot{a}^{2}\left(1+\frac{4 a L}{3 R_{T}^{2}}\right)=\Delta P . \\
& \Delta P=P_{0}\left(\frac{a_{0}}{a}\right)^{3 \kappa}-P_{0}-\frac{4 \eta_{L} \dot{a}}{a}-\frac{4 \kappa_{S} a_{0}^{2} \dot{a}}{a^{4}}-\frac{4 \chi a_{0}^{2}}{a^{3}}\left(1-\frac{a_{0}}{a}\right)-P_{\mathrm{US}}(t) .
\end{aligned}
$$

The symbols $L$ and $R_{T}$ stand for the tube length and the tube radius, respectively. $\kappa_{S}$ is the shell dilatational viscosity and $\chi$ its elastic compression modulus. The assumption of a rigid tube with fixed tube radius $R_{T}$ is permissible due to the highly indistensible nature of capillary vessels: a variation in $R_{T}$ of the order of a few per cent as observed in our simulations was found to have no relevant impact on the transient $\mathrm{MB}$ radius.

Most modern UCAs have lipid shells (Kaul 2008), an equilibrium diameter of 2-3 $\mu \mathrm{m}$ (Greis 2004, Sontum 2008, Faez et al 2011) and a shell parameter range for $\kappa_{S}$ of $5-16 \mathrm{nN} \mathrm{s} \mathrm{m}^{-1}$ and for $\chi$ of $0.6-1 \mathrm{~N} \mathrm{~m}^{-1}$ (Goertz et al 2007, Faez et al 2011, Marmottant et al 2005). The shell parameters in this study are chosen as $\kappa_{S}=15 \mathrm{nN} \mathrm{s} \mathrm{m}^{-1}$ and $\chi=1 \mathrm{~N} \mathrm{~m}^{-1}$ with $a_{0}$ ranging from 2 to $3 \mu \mathrm{m}$.

Equations (3) and (4) may be combined to produce an ordinary differential equation (ODE) which describes the comportment of an encapsulated MB with a lipid monolayer coating inside a confining tube. This ODE is solved at run-time in OpenFOAM using a fifth-order Runge-Kutta algorithm (Cash and Karp 1990) to obtain the instantaneous bubble radius. The computational domain is modified at each time step with the above mentioned grid adaptation algorithm to accommodate the new bubble size.

\subsection{Liquid domain model}

Blood plasma inside the compliant vessel is modelled as a homogeneous, single phase, isothermal, incompressible Newtonian fluid with constant dynamic viscosity of $\eta_{L}=1.5 \mathrm{mPa} s$ (Rand et al 1964, Lowe 1987, Haidekker et al 2002) and density of $\rho_{L}=1030 \mathrm{~kg} \mathrm{~m}^{-3}$ (Hinghofer-Szalkay and Greenleaf 1987, Lowe 1987, Stride 2005). The flow of liquid in an arbitrary volume $V$ bounded by a moving surface $S$ is governed by the mass and linear momentum conservation laws:

$$
\begin{aligned}
& \int_{S} \mathbf{n} \cdot \mathbf{v} \mathrm{d} S=0 \\
& \frac{\mathrm{d}}{\mathrm{d} t} \int_{V} \mathbf{v} \mathrm{d} V+\int_{S} \mathbf{n} \cdot\left(\mathbf{v}-\mathbf{v}_{S}\right) \mathbf{v} \mathrm{d} S=\int_{S} \mathbf{n} \cdot\left(v_{L} \nabla \mathbf{v}\right) \mathrm{d} S-\frac{1}{\rho_{L}} \int_{V} \nabla P \mathrm{~d} V,
\end{aligned}
$$

where $\mathbf{n}$ is the outward pointing unit normal on $S, \mathbf{v}$ is the liquid velocity, $\mathbf{v}_{S}$ is the displacement velocity of the surface $S, v_{L}=\eta_{L} / \rho_{L}$ is the kinematic viscosity of the liquid and $P$ is pressure. 
The relationship between the rate of change of the volume $V$ and the velocity $\mathbf{v}_{s}$ is defined by the space conservation law (Demirdzic and Peric 1988):

$$
\frac{\mathrm{d}}{\mathrm{d} t} \int_{V} \mathrm{~d} V-\int_{S} \mathbf{n} \cdot \mathbf{v}_{S} \mathrm{~d} S=0
$$

The governing equations written in arbitrary Lagrangian-Eulerian (ALE) formulation are discretized in space using a second-order accurate cell-centred unstructured finite volume (FV) method. The resulting algebraic system is solved using a segregated solution procedure based on the PISO algorithm (Issa 1986) for the pressure-velocity coupling.

The transient problem is solved in a time-marching manner along the discretized time direction with uniform time steps of size $\Delta t$. An implicit second-order accurate three-time-level scheme (Ferziger and Peric 1995) is employed for the temporal discretization of the model. Full details on the numerical methods used can be found in Tuković (2005) and Tuković and Jasak (2011).

\subsection{Red blood cells}

RBCs are located to either axial side of the MB (figure 1). RBCs and MBs show similar kinetics of circulation (Jayaweera et al 1994); statistical mixing of the two species can thus be assumed. Comparing the concentration of RBCs in human blood (McHedlishvili and Varazashvili 1980) to commonly used concentrations of MBs in solution (Tu et al 2009) reveals that a single MB inside a typical capillary will be surrounded by some $10^{2} \mathrm{RBCs}$ on either side with a distance of 5-7 $\mu \mathrm{m}$ to the closest RBC. Consequently, the MB under investigation will be well isolated from other oscillating MBs in terms of fluid dynamics.

The RBCs are modelled as homogeneous, isotropic, linear elastic solids that may freely move along the tube symmetry axis. Their density is prescribed as $\rho_{R}=1110 \mathrm{~kg} \mathrm{~m}^{-3}$ (Grover et al 2011, Godin et al 2007, Hinghofer-Szalkay and Moser 1986). Young's modulus $E_{R}$ is assumed to be similar to that of the vessel wall, which is considerably higher than the reported range of 20-40 kPa for RBCs in the relaxed state (Zuk et al 2011, Dulinska et al 2006). Our choice of the higher modulus is motivated by the fact that the modelled RBCs are not in relaxed states, and that their radial deformation is limited by the vessel stiffness. In addition, there are no published data on the mechanical properties of RBCs in the given deformed state.

\subsection{Vessel structure}

The capillary endothelial layer together with the associated basal membrane is treated as a distinct solid domain encompassing the fluid domain. It is modelled using homogeneous, isotropic and linear elastic material properties: density $\rho$ of $1200 \mathrm{~kg} \mathrm{~m}^{-3}$, Poisson's ratio $v_{P}$ of 0.45 and Young's modulus $E$ between 1 and $10 \mathrm{MPa}$ (Miao et al 2008). The rigidity of a capillary vessel is determined by the compound of endothelium, basement membrane and the surrounding tissue (Murphy and Johnson 1975). Capillaries are typically almost indistensible with distensibility:

$$
\beta=\frac{1}{D_{0}}\left(\frac{\mathrm{d} D}{\mathrm{~d} P}\right)
$$

of the order of $10^{-3} \mathrm{mmHg}^{-1}$ (Baldwin and Gore 1989). Here, $D$ is the current vessel diameter and $D_{0}$ is a reference vessel diameter. The vessel stiffness is dependent on its location within the body as well as the age of the subject (Zieman et al 2005) and differs in tumours compared to healthy tissue (Qin and Ferrara 2006). 


\subsection{Solid domain model}

The dynamic behaviour of the solid domains (vessel wall and RBCs) is described by the momentum conservation law shown in equation (9), where it is written in an incremental formulation that takes only differences between quantities at the immediately preceding and the current time step into account. The previous time step configuration is assigned as reference frame for the employed updated Lagrangian formulation.

$$
\int_{V_{u}} \rho_{u} \frac{\partial}{\partial t}\left(\frac{\partial \delta \mathbf{u}}{\partial t}\right) \mathrm{d} V_{u}=\int_{S_{u}} \mathbf{n}_{u} \cdot\left(\delta \boldsymbol{\Sigma}_{u}+\boldsymbol{\Sigma}_{u} \cdot \delta \mathbf{F}_{u}^{\mathrm{T}}+\delta \boldsymbol{\Sigma}_{u} \cdot \delta \mathbf{F}_{u}^{\mathrm{T}}\right) \mathrm{d} S_{u} .
$$

Here, $\mathbf{u}$ is the displacement vector, $\boldsymbol{\Sigma}$ is the second Piola-Kirchhoff stress tensor and $\mathbf{F}=\mathbf{I}+(\nabla \mathbf{u})^{\mathrm{T}}$ is the deformation gradient tensor. The subscript $u$ indicates quantities related to the updated Lagrangian reference configuration which is the last calculated configuration. The operator $\delta(\cdot)$ gives the increment of a variable between the old time instance $t^{\circ}$ and the new time instance $t^{n}=t^{o}+\Delta t$.

The solid's stress-strain relationship is defined by the Saint Venant-Kirchhoff constitutive model, which in updated Lagrangian incremental formulation reads

$\delta \boldsymbol{\Sigma}_{u}=\mu\left[\nabla \delta \mathbf{u}+(\nabla \delta \mathbf{u})^{\mathrm{T}}\right]+\lambda \operatorname{tr}(\nabla \delta \mathbf{u}) \mathbf{I}+\mu \nabla \delta \mathbf{u} \cdot(\nabla \delta \mathbf{u})^{\mathrm{T}}+\frac{1}{2} \lambda(\nabla \delta \mathbf{u}: \nabla \delta \mathbf{u}) \mathbf{I}$,

where $\mu$ and $\lambda$ are Lamé's coefficients calculated from $E$ and $\nu_{P}$ and the double dot operator denotes a double inner product.

The spatial and temporal discretization of the model is performed similar to the case of the fluid domain (see section 2.5) using a second-order cell-centred FV method in space and an implicit second-order accurate three-time-level scheme for time. The solution is obtained using a segregated procedure (Tuković and Jasak 2007, Maneeratana 2000).

\subsection{Coupling of the domains}

The implementation of the dynamic interaction of the three physically distinct domains relies on the transfer of relevant quantities across their common interfaces. A solution at the interface of one domain will be translated into boundary conditions for the adjacent domain and vice versa. This partitioned approach offers flexibility in the choice of the mathematical models used to represent each individual domain and allows for the utilization of specialized solvers.

The role of the MB as an explicit dynamic source is accounted for by a one-way coupling to the liquid (figure 3). A change in radius $\delta a$ derived from the modified RPE is translated into an adaptation of the liquid mesh configuration to accommodate the new MB shape. This geometrical alteration induces a flow which in turn affects the adjacent solid domain.

The interaction between liquid flow and solid dynamics is treated by means of a twoway coupling to account for the strong mutual influence of blood plasma and the compliant capillary vessel or RBCs. Figure 3 shows that interface pressure increment $\delta p_{I}$ and interface traction increment $\delta \mathbf{t}_{I}$ values from the liquid side are used to determine the interface boundary conditions for the solid model. The resulting interface displacement increment $\delta \mathbf{u}_{I}$ is employed in turn to deform the liquid domain by means of the mesh motion solver. The interface displacement velocity $\mathbf{v}_{I}$ is integrated into the liquid boundary condition in the scope of the ALE formulation. This algorithm works as a segregated iterative process where the flow solver and the solid solver are used consecutively with the aim of achieving momentum equilibrium at every time step of the transient simulation.

In order to manage the very tight coupling between the two domains, an IQNILS (interface quasi-Newton technique with inverse Jacobian from a least-squares model) coupling algorithm (Degroote et al 2009) was introduced to obtain higher stability and faster convergence of the solution process. 


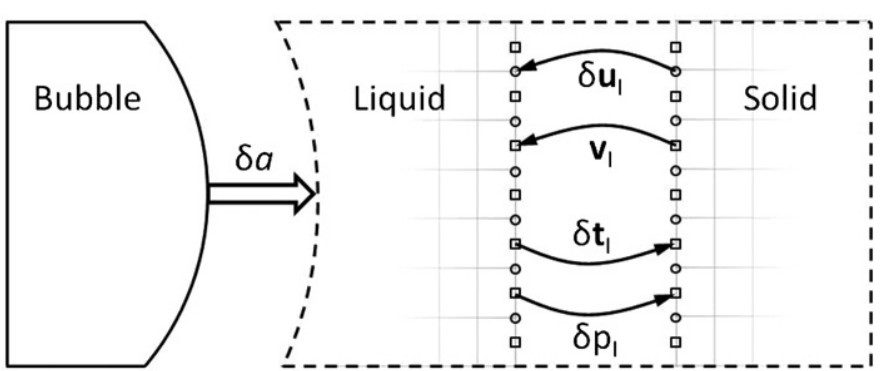

Figure 3. Schematic of the overall working principle of the coupling framework. The bubble, described by a modified RPE, acts as the driving force for the dynamics of the system. It agitates the liquid by displacing the bubble-liquid interface by $\delta a$. The induced microflow impacts the compliant solid components, which is accounted for by transferring interface pressure increment $\delta p_{I}$ and interface traction increment $\delta \boldsymbol{t}_{I}$ from the liquid side across the liquid-solid interface and incorporating them into the solid's boundary conditions. Consecutively, the position of the liquidsolid interface is changed by the interface displacement increment $\delta \boldsymbol{u}_{I}$ derived from the solid equations and the displacement velocity $\boldsymbol{v}_{I}$ is incorporated into the liquid's boundary conditions. This liquid-solid coupling cycle is repeated until momentum equilibrium is reached.

\subsection{Boundary conditions and initial conditions}

The average absolute intraluminal pressure in a capillary is approximately $105 \mathrm{kPa}$ (Qin and Ferrara 2007), corresponding to an average overpressure relative to the perivascular space of 2.6 to $4 \mathrm{kPa}$ (Eichna 1942, Sassaroli and Hynynen 2004). This initial condition is put into place in a preconditioning step by inflating the initially stress-free compliant vessel to the appropriate pressure level of $3.3 \mathrm{kPa}$, thereby loading the configuration.

Because of the incompressible nature of the liquid, only pressure differences are of relevance. Therefore, the extravascular pressure level $P_{e}$, which accounts for the constant pressure exerted on the vessel by interstitial fluid and surrounding tissue, is set to zero. This reference renders the intraluminal pressure $P_{i}(r, z)$ equivalent to the local overpressure.

The time-varying incident ultrasound pressure field $P_{\mathrm{US}}(t)$, taken to be homogeneous throughout the domain (see section 2.1), is also subtracted from the overall pressure. This measure may be introduced because the influence of the ultrasound field on the dynamics of the system is accounted for by the action of the MB with $P_{\mathrm{US}}(t)$ incorporated in the modified RPE.

Following the line of thought of Qin and Ferrara (2006) and Martynov et al (2009), we consider fixed pressure boundary conditions at the vessel inlet. A free traction boundary condition is applied to the outer vessel surface, a symmetry boundary condition is assigned to the central cross-section and axisymmetry boundary conditions are employed on the wedge surfaces.

\section{Results}

We have used the described modelling framework to investigate the flow patterns and mechanical wall conditions for our model vessel with MBs of $a_{0}=1.0 \mu \mathrm{m}$ (case 1) and $a_{0}=1.3 \mu \mathrm{m}$ (case 2). The results are compared to those of cases without RBCs and with rigid, spatially fixed RBCs which represent limiting configurations. The vessel wall stiffness is treated as a parameter to investigate its influence on pressure and shear stress. The reported results are independent of the spatial and temporal discretization (see supplemental material available at stacks.iop.org/PMB/57/1019/mmedia). 

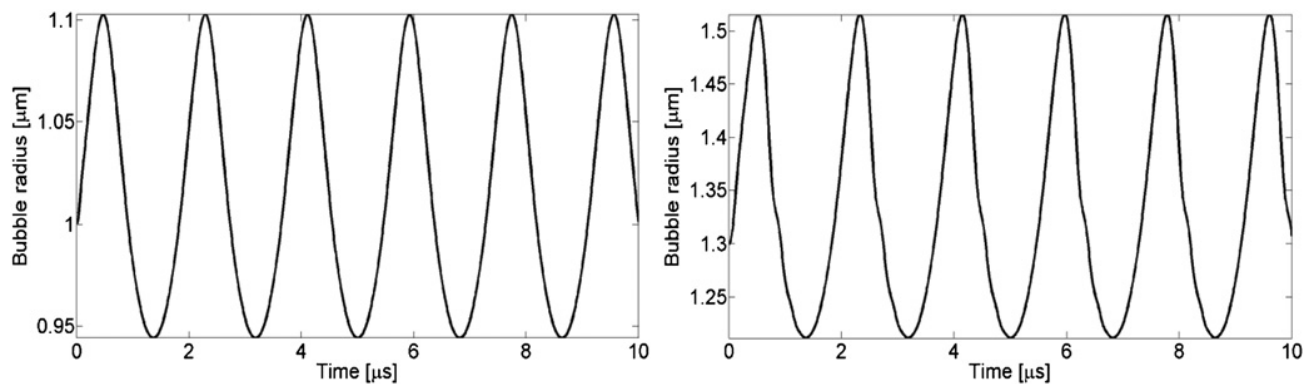

Figure 4. Bubble radius versus time curves derived from the modified RPE for the two considered cases with equilibrium radius $a_{0}=1.0 \mu \mathrm{m}$ (left) and $a_{0}=1.3 \mu \mathrm{m}$ (right). Both bubbles are encapsulated with a shell characterized by $\kappa_{S}=15 \mathrm{nN} \mathrm{s} \mathrm{m}^{-1}$ and $\chi=1 \mathrm{~N} \mathrm{~m}^{-1}$, and confined in a capillary vessel of $6 \mu \mathrm{m}$ diameter.

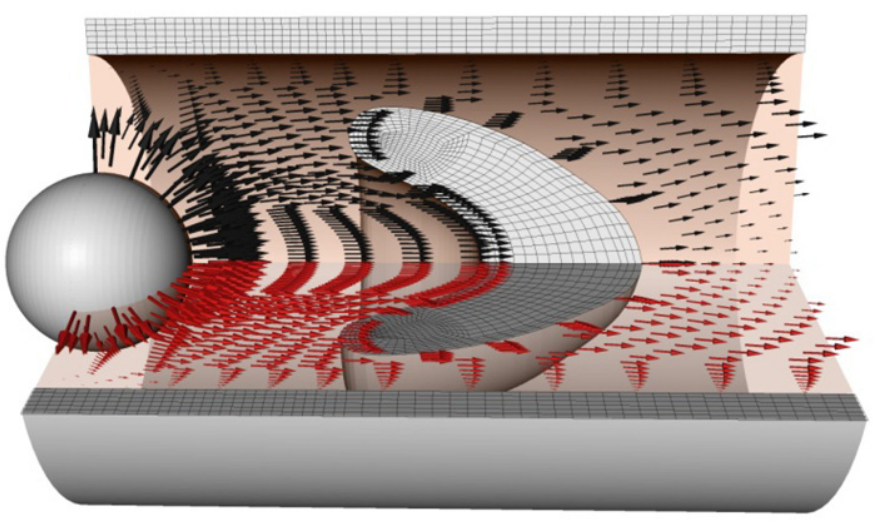

Figure 5. Flow pattern for case 2 during MB expansion. Flow velocities are highest at the bubble surface and show a rapid drop in radial direction as a consequence of the three-dimensional nature of the setup. The flow is laminar and a shear flow layer near the wall is clearly visible. The colouring of the velocity vectors serves to illustrate their affiliation with the different cutting planes.

The respective curves of bubble radius versus time as derived from the modified RPE (3) and (4) are shown in figure 4. A predominantly regular and uniform oscillation pattern emerges when the influence of the bubble shell is taken into account. This behaviour is in accordance with Stride and Saffari (2003).

\subsection{Flow properties}

The oscillatory flow induced by the volumetric expansion and contraction of the MB is characterized by peak bubble surface velocities between $0.33 \mathrm{~m} \mathrm{~s}^{-1}$ (case 1) and $0.96 \mathrm{~m} \mathrm{~s}^{-1}$ (case 2). Maximum near wall flow speeds range from 0.1 to $0.31 \mathrm{~m} \mathrm{~s}^{-1}$. A clearly visible shear layer is established along the vessel wall starting at some distance from the $\mathrm{MB}$ (figure 5). Comparison to a setup without $\mathrm{RBC}$ reveals that the velocity field is almost undisturbed by the presence of the RBC during the $\mathrm{MB}$ expansion and contraction phases. The impact of the RBC becomes apparent during the transition between expansion and contraction phases, when the flow is reversed and the RBC's inertia delays the process (figure 6). 


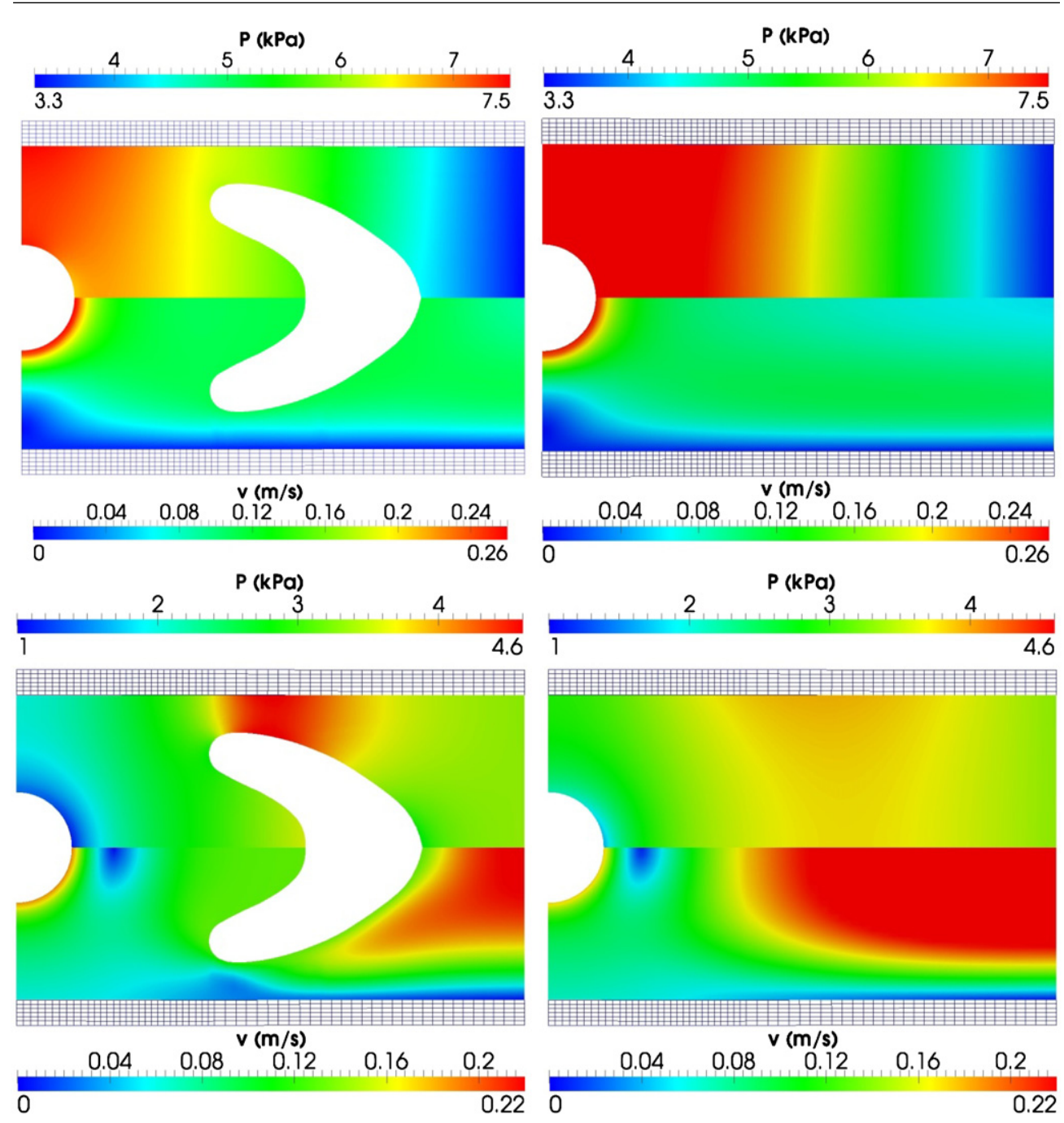

Figure 6. Pressure (upper vessel section) and velocity distribution (lower vessel section) for case 1 (left column) and the respective configuration without RBC (right column). The top row compares conditions during the expansion phase, where the presence of the RBC has little impact on the pressure distribution and almost no impact on the flow field. The bottom row depicts the same comparison at the onset of the contraction phase when the flow is reversed. Here, the flow field is significantly altered due to the inertia of the RBC and the pressure distribution is less homogeneous than without RBC.

The RBCs are free to move in axial direction. Their displacement amplitudes are $0.08 \mu \mathrm{m}$ (case 1) and $0.18 \mu \mathrm{m}$ (case 2). These small values are conceivable despite the relatively high velocities, as the very small time scale of oscillation renders the overall fluid flux small.

The intraluminal pressure level $P_{i}$ as defined in section 2.10 varies between -2 and $8.3 \mathrm{kPa}$ (case 1), and -17.5 and $17.2 \mathrm{kPa}$ (case 2). The pressure distribution induced by the $\mathrm{MB}$ is mostly unaffected by the presence of the RBC, with the exception of the phases where the $\mathrm{MB}$ is close to its maximum and minimum radius, respectively, as can be seen by comparison to a case without RBC (figure 6). 

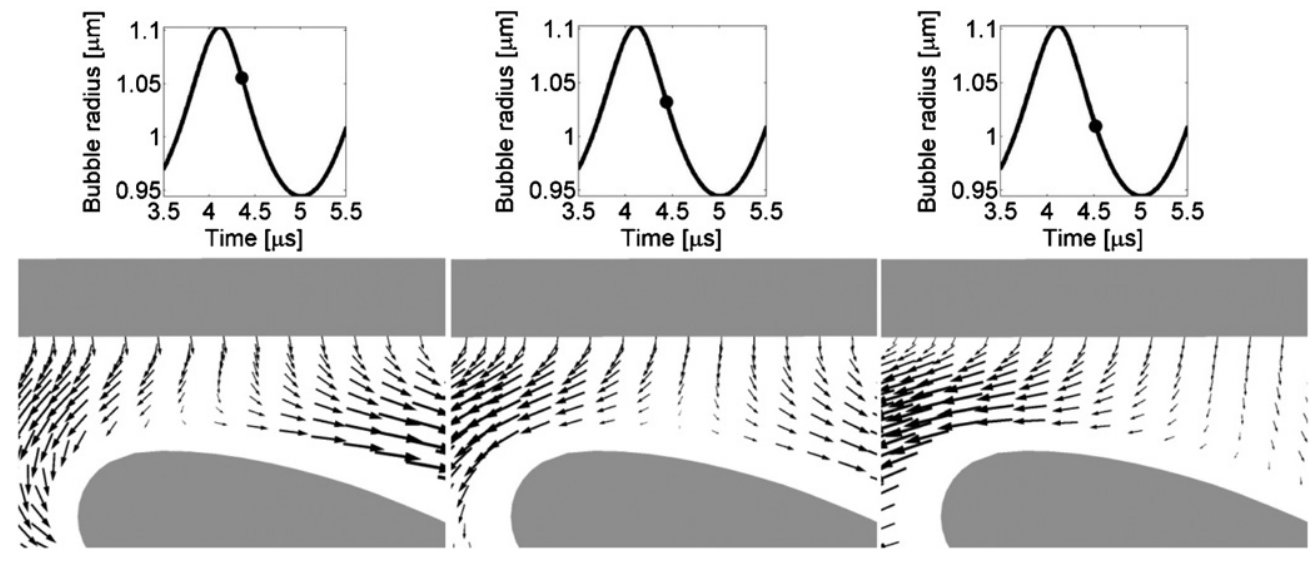

Figure 7. Consecutive stages of the flow splitting in the passage between the rim of the RBC (grey area at the bottom) and the vessel wall (grey area at the top) during the initial phase of MB contraction in case 1 . The graphs on top of the respective plots show the current radius configuration using a phase point on the radius-time curve derived from the modified RPE. This behaviour is observed in all investigated cases with mobile as well as fixed RBCs.
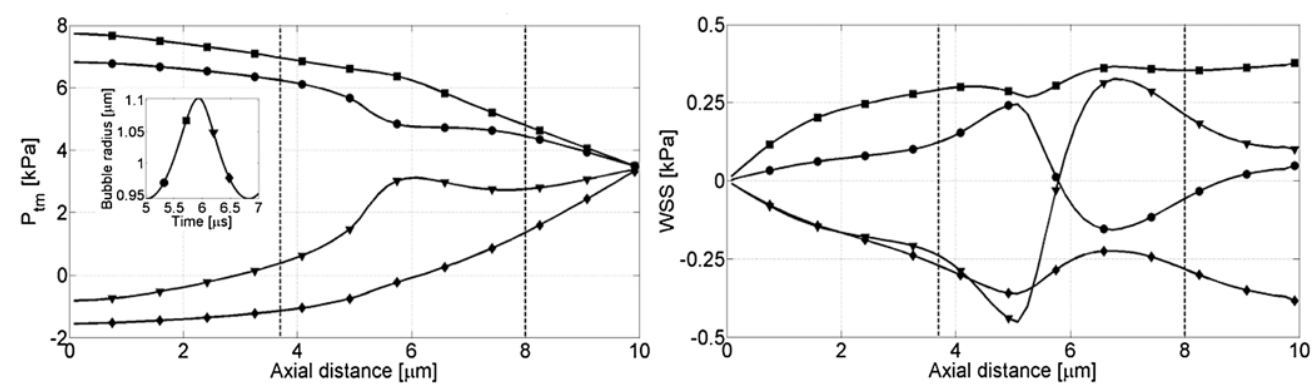

Figure 8. Data for case 1: transmural pressure (left) and WSS distribution (right) along the vessel wall in axial direction starting at the location closest to the MB centre $(z=0)$ for four instances in time representative of the MB expansion and contraction phases. The MB radii at those times are visualized in the inset and the respective markers are used in the figures for $P_{\mathrm{tm}}$ and WSS. Values are independent of the azimuthal angle due to the cylindrical symmetry of the setup. The axial location of the RBC is indicated by the dashed vertical lines. The strongest disturbances are observed during the onset of expansion (circles) and contraction (triangles) in the vicinity of the RBC.

While the flow pattern is orderly throughout the better part of the oscillation cycle, a temporary splitting of flow into two branches may be observed in the early phase of the rapid $\mathrm{MB}$ contraction (figure 7). The splitting line separating the two jets is generated close to the rim of the RBC and moves towards the distal region in time. This effect leads to retarded reflux of blood plasma from the distal compartment towards the contracting MB.

Similarly, a converging flow pattern is found in the vicinity of the RBC in the early expansion phase of the MB. Both effects become apparent in the patterns of the WSS (figures 8 and 9) and its spatial derivative (figure 10). They are equally observed in case 2 as well as in the configurations with fixed and rigid RBC. 

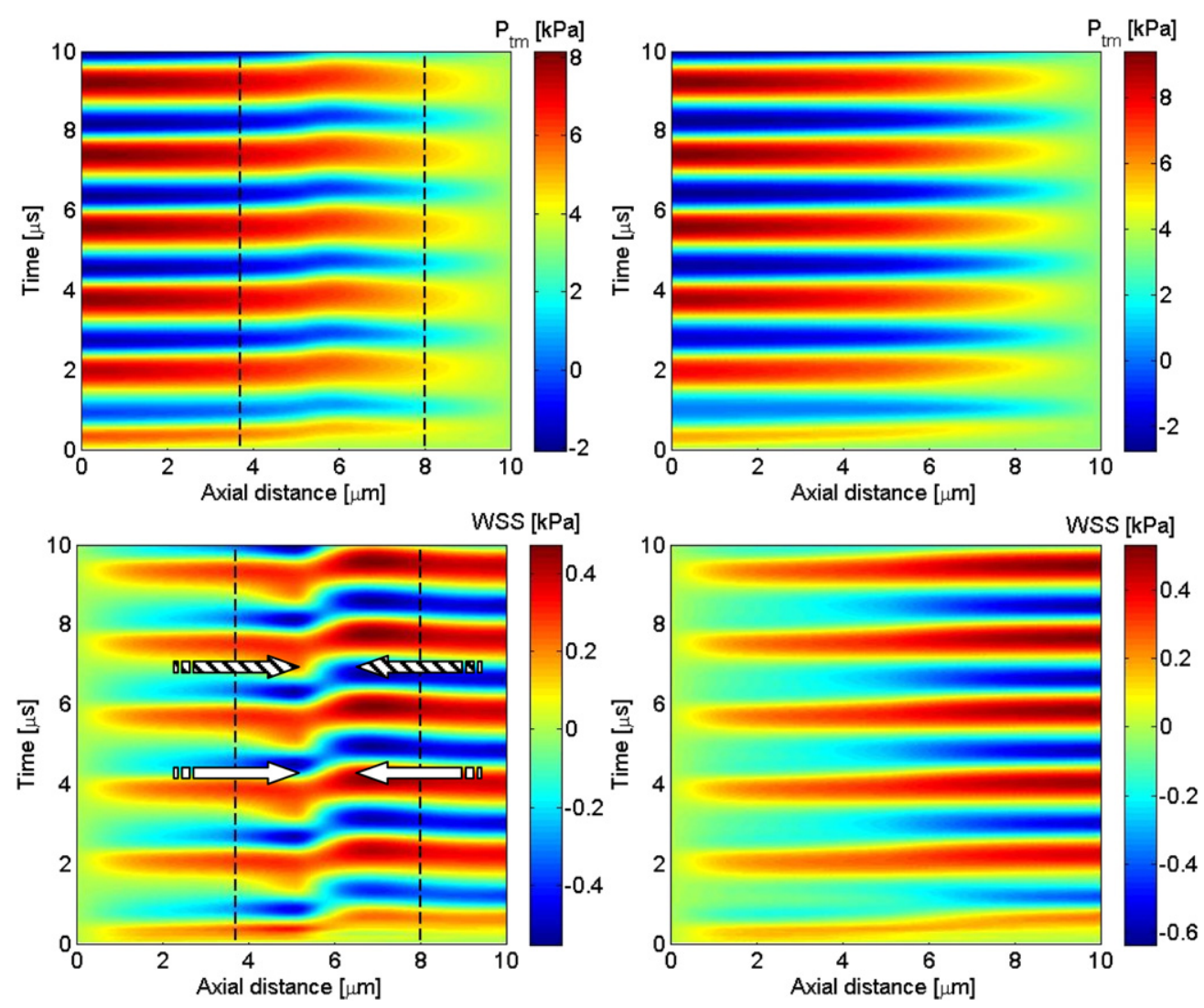

Figure 9. Mechanical conditions at the vessel interface with $E=8 \mathrm{MPa}$ for case 1 (left column) compared to the respective configuration without RBC (right column): temporal development of the transmural pressure (top) and WSS (bottom) along the vessel wall in axial direction. The dashed lines mark the projected location of the RBC. The patterns are almost identically repetitive in time. The presence of the RBC causes a slight buckle in the pressure pattern and a considerable disturbance in the spatial WSS pattern in the vicinity of the RBC. The white arrows highlight a representative state of flow splitting near the $\mathrm{RBC}$ in the $\mathrm{MB}$ contraction phase, where one portion of the liquid has negative axial velocity components and the more distal portion has positive components. The corresponding WSS values thus have different signs (negative and positive). Similarly, the opposite state of a converging flow is highlighted with shaded arrows.

\subsection{Endothelial interface conditions}

The mechanical conditions at the luminal endothelial surface are the focus of this study, since they are suspected to play a key role in BBBD. The current position of this interface is given by $r=R_{I}(z)$. Of particular interest are the transmural pressure,

$$
P_{\mathrm{tm}}(z)=P_{i}\left(R_{I}, z\right)-P_{e},
$$

defined as the difference between the pressure at the luminal wall interface $P_{i}\left(R_{I}, z\right)$ and the pressure in the perivascular space outside the capillary $P_{e}$ (see section 2.10), and WSS which is calculated for each interface element as

$$
\mathrm{WSS}=\left.\eta_{L} \frac{\mathrm{dv}_{\|}}{\mathrm{d} n}\right|_{r=R_{I}} .
$$



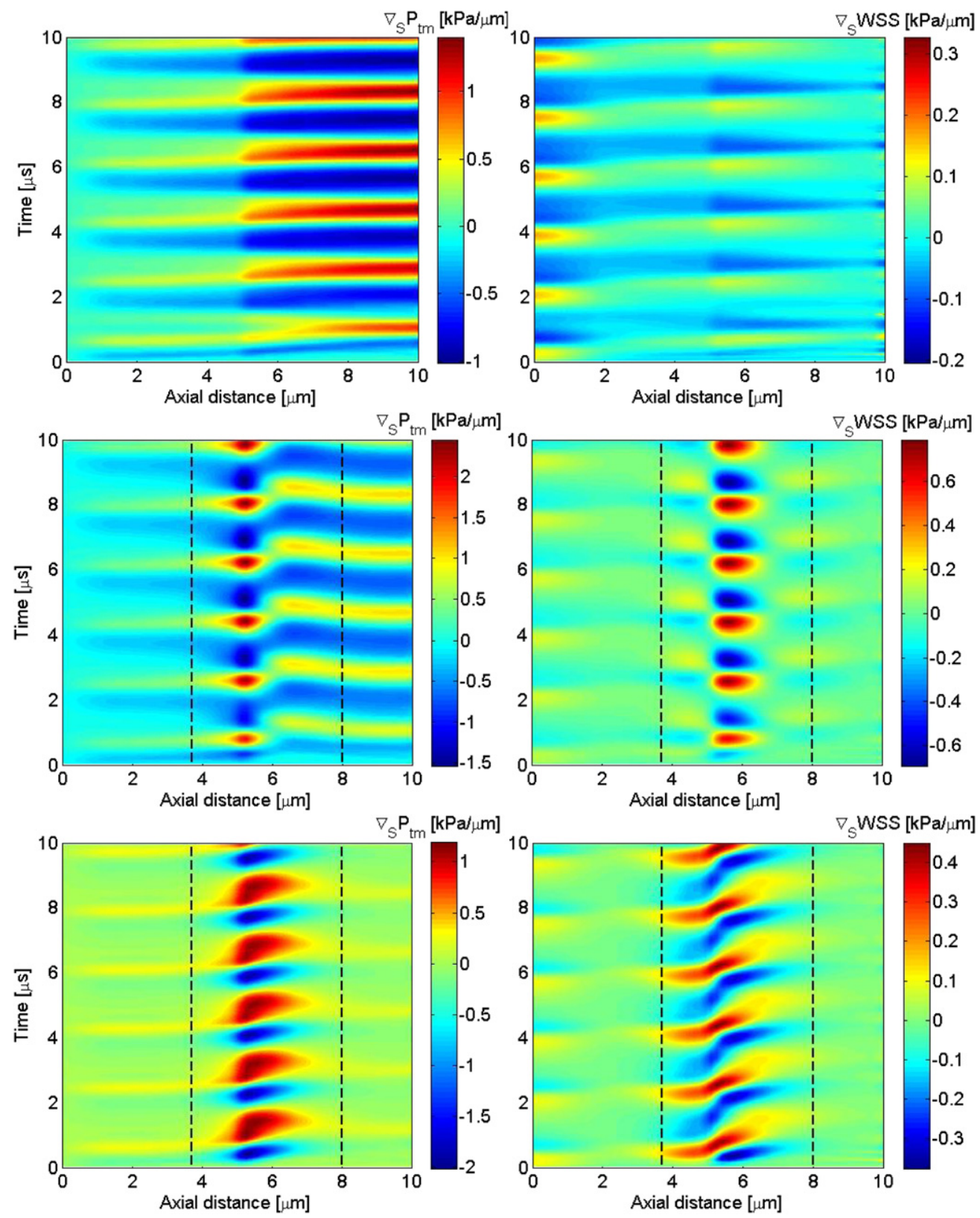

Figure 10. Spatial surface derivatives of wall parameters at $E=8 \mathrm{MPa} . P_{\mathrm{tm}}$ (left column) and WSS (right column) for case 1 (middle row), the respective configuration without RBC (top row), and the configuration with rigid and fixed RBC (bottom row). For the case without RBC, the spatial gWSS gradient is rather uniform, while pronounced positive and negative peaks occur in the presence of an $\mathrm{RBC}$. The spatial $\mathrm{g} P_{\mathrm{tm}}$ gradient gradually increases towards the inlet for a setup without RBC, while it again shows pronounced peaks in the vicinity of RBCs. It is remarkable that for both parameters the highest values and the spatially most compact peaks are attained in the setup with a flexible, mobile RBC. 
Here $\mathrm{v}_{\|}$is the component of liquid velocity parallel to the wall and $n$ is the direction normal to the vessel wall pointing into the vessel lumen. While in general WSS is a vector in the tangential plane of the considered wall element, it reduces here to a scalar quantity due to the axial symmetry of the considered setup.

$P_{\mathrm{tm}}$ and WSS were recorded at each time step of the transient simulation along the length of the vessel section; the cylindrical symmetry renders them independent of the azimuthal angle. Figure 8 shows transmural pressure and WSS distributions at four representative instances during the expansion and contraction phases of the MB for case 1.

It is remarkable that a kink in the $P_{\mathrm{tm}}$ distribution appears during the initial phases of $\mathrm{MB}$ contraction and expansion. At the same time, strong gradients in the WSS field are observed at roughly the same locations. WSS changes signs in those situations, which is an indicator for flow splitting (positive gradient) or converging flow (negative gradient). Flow splitting is characterized by negative proximal WSS values, indicating flow in the negative $z$-direction, while more distal regions carry the opposite sign.

A more complete impression of the transient process can be gained by plotting the distribution of $P_{\mathrm{tm}}$ and WSS along the length of the vessel section over time using contour plots (figure 9). The resulting pattern represents the temporal development of the distribution of a mechanical parameter along the vessel wall. The location of the RBC along the vessel axis is indicated by dashed lines.

For case 1, the patterns repeat themselves in time owing to the regular, rather uniform radius function (figure 4 ). The pressure distribution looks very similar to the pattern without $\mathrm{RBC}$, but shows a small shift towards higher time values in the central RBC region. This corresponds to the appearance of a kink in the $P_{\mathrm{tm}}$ patterns identified in figure 8 . Increased spatial $P_{\mathrm{tm}}$ gradients $\left(\mathrm{g} P_{\mathrm{tm}}\right)$ may be expected at these locations.

The WSS patterns are significantly disturbed by the presence of the RBC. At certain instances, the shear stress changes sign, which indicates either a state of flow splitting or converging flow. These flow configurations lead to substantial spatial WSS surface gradients (gWSS). The location of the splitting line or merging line between the two jets corresponds approximately to the position of the zero WSS region separating the WSS extrema. Figure 10 shows the corresponding gWSS distribution and reveals that the splitting line position exhibits peak gradient readings. WSS vanishes at the zero axial location, because planar symmetry only permits radial flow there. The maximum absolute WSS values gathered are remarkably high, ranging up to $0.42 \mathrm{kPa}$, which is about two orders of magnitude above physiological values of 2-10 $\mathrm{Pa}$ (Reneman et al 2006).

The appearance of localized peaks of spatial surface gradients seems to be an intriguing feature of a configuration containing RBCs. A comparison of the spatial gradients for $P_{\mathrm{tm}}$ and WSS of case 1 with the corresponding cases without RBC and with a rigid and fixed RBC is shown in figure 10. The configuration without RBC demonstrates a rather homogeneous variation of gWSS while there are localized positive and negative peaks visible in the presence of the RBC. The case with mobile and flexible RBC shows a more compact concentration with notably higher values. With view of $\mathrm{g} P_{\mathrm{tm}}$ a monotonous characteristic is visible for the case without RBC, while localized peaks appear in the presence of RBCs. Again, the region of peak values is more compact in the case of a mobile and flexible RBC and the absolute values are notably higher. Thus, while the introduction of a mobile and flexible RBC has small to moderate impact on the $P_{\mathrm{tm}}$ and WSS patterns, it seems to alter the gradient patterns markedly.

Figure 11 shows the transient pressure and WSS patterns for case 2, which features a $30 \%$ larger $\mathrm{MB}$ equilibrium radius compared to case 1. Oscillations are again regular (figure 4), resulting in very similar mechanical patterns involving the same features such as a kink in the pressure distribution and a disturbance of the WSS field due to the presence of 


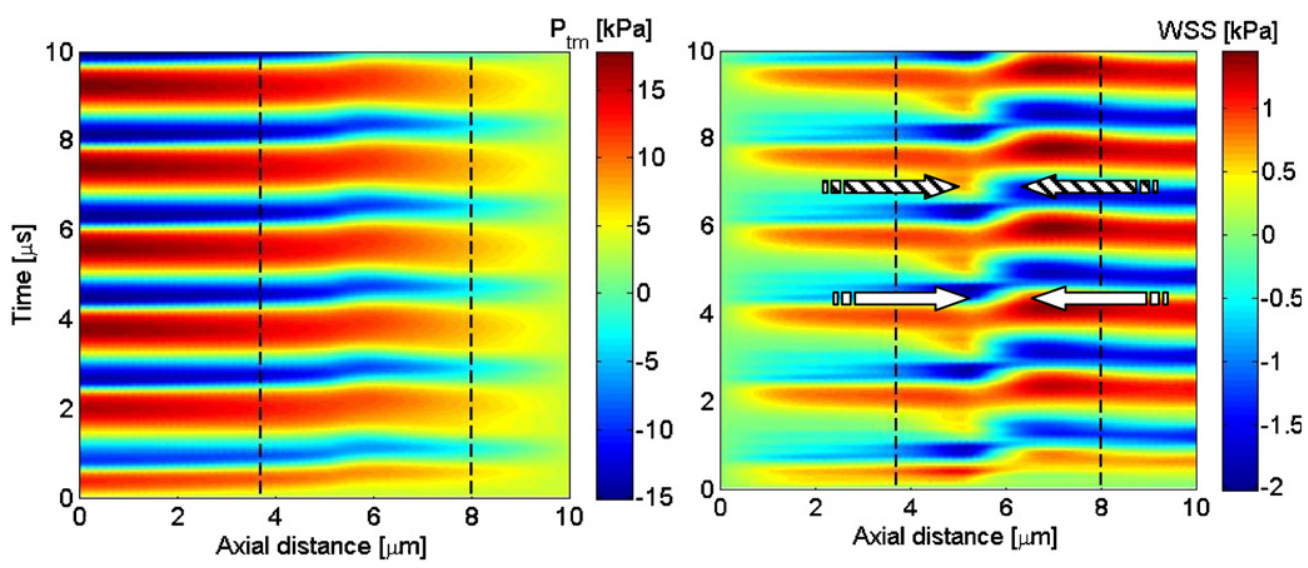

Figure 11. Data for case 2 with $E=8 \mathrm{MPa}$ showing temporal development of the transmural pressure (left) and WSS (right) distribution along the vessel wall. The dashed lines indicate the location of the RBC. The patterns including the flow splitting (highlighted by the white arrows) and converging flow regions (highlighted by the hatched arrows) match basically those of case 1 but show a significant increase in absolute values.

the RBC. States of flow splitting and converging flow in the vicinity of the RBC can also be identified. However, the maximum values for $P_{\mathrm{tm}}$ and WSS are considerably higher than in case 1 . The same is true for their spatial derivatives.

The peak absolute values for $P_{\mathrm{tm}}$ and WSS as well as for their spatial gradients are compared in figure 12, contrasting them with results for the setup without RBC and with a rigid and fixed RBC. It is remarkable that the pressure readings are highest for the setup without RBC and lowest for the setup with stationary RBC. The peak spatial gradients in the setup with a flexible and mobile RBC clearly surpass the values for the other configurations, which is also visible in figure 10.

\subsection{Variation of vessel rigidity}

The rigidity of individual capillary vessels may vary significantly and is only known by order of magnitude (see section 2.7). We have thus treated Young's modulus of the solid domain as a parameter and checked for its impact on the transmural pressure and shear stress conditions. Values of 4 to $9 \mathrm{MPa}$ were used in otherwise identical case setups, and the maximum occurring overall WSS and $P_{\mathrm{tm}}$ values were compared as shown in figure 13 for case 1 . The relative increase due to the variation in Young's modulus is monitored: a doubling of $E$ leads to an increase of roughly $65 \%$ in peak absolute WSS and a $40 \%$ augmentation of peak absolute transmural pressure.

An increase of the vessel rigidity not only causes a change in absolute values of $P_{\mathrm{tm}}$ and WSS values, but also alters their patterns. Comparing the results for $E$ of 4 and $9 \mathrm{MPa}$, a shift in the temporal occurrence of $P_{\mathrm{tm}}$ peak can be seen (figure 14). This observation also holds for the WSS pattern in the distal compartment, where regions of similar WSS seem to become almost disconnected in the more compliant vessel. The more flexible configuration at $E=$ $4 \mathrm{MPa}$ appears to delay onset of peak $P_{\mathrm{tm}}$ and peak distal WSS, which may be attributed to the higher degree of compliance. The slight kink in the pressure distribution caused by the presence of the RBC seems more pronounced in the more compliant vessel. A shift of the peak WSS values from the vicinity of the proximal rim of the RBC at $E=4 \mathrm{MPa}$ to the more distal position is seen. 

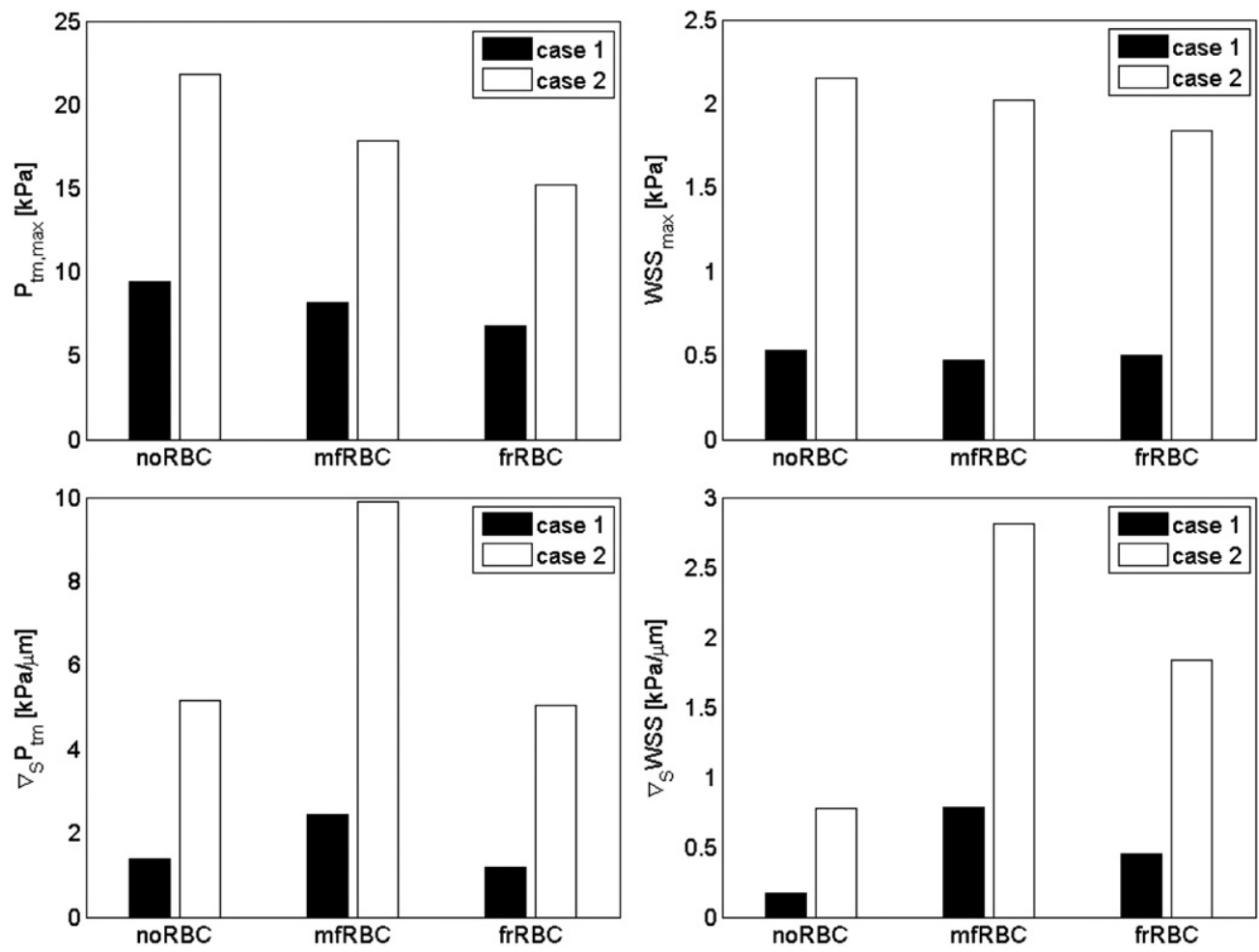

Figure 12. Comparison of the peak absolute values for $P_{\mathrm{tm}}$ and WSS as well as their spatial derivatives of case 1 and case 2 with $E=8 \mathrm{MPa}$ for the configuration without RBC (noRBC), the standard configuration with mobile and flexible RBC (mfRBC) and the configuration with fixed and rigid RBC (frRBC). It is remarkable that the $P_{\mathrm{tm}}$ and WSS readings are highest for the setup without $\mathrm{RBC}$ and lowest for the setup with stationary $\mathrm{RBC}$. Conversely, the highest $\mathrm{g} P_{\mathrm{tm}}$ and gWSS values are found for the configuration with mobile and flexible RBC.

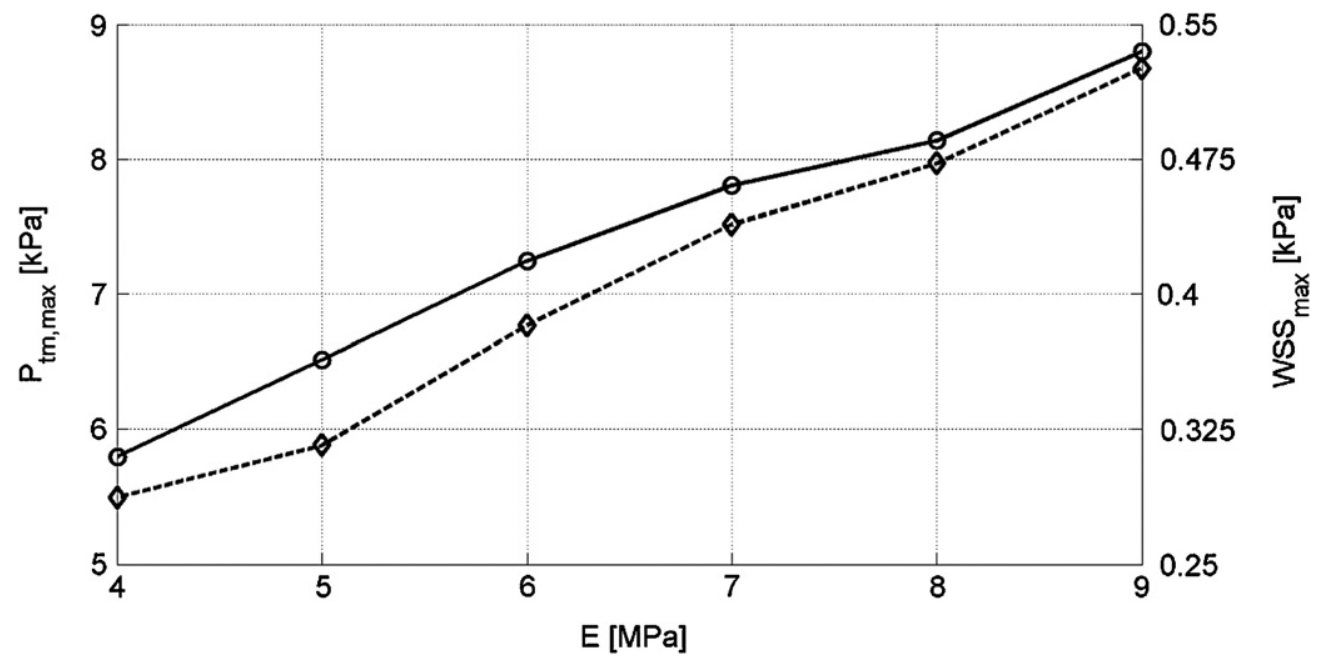

Figure 13. Variation of overall peak transmural pressure (solid line) and peak WSS (dashed line) as a function of Young's modulus of the vessel wall for case 1. While maximum WSS increases by around $65 \%$ when Young's modulus is doubled, the maximum transmural pressure increases by approximately $40 \%$. 

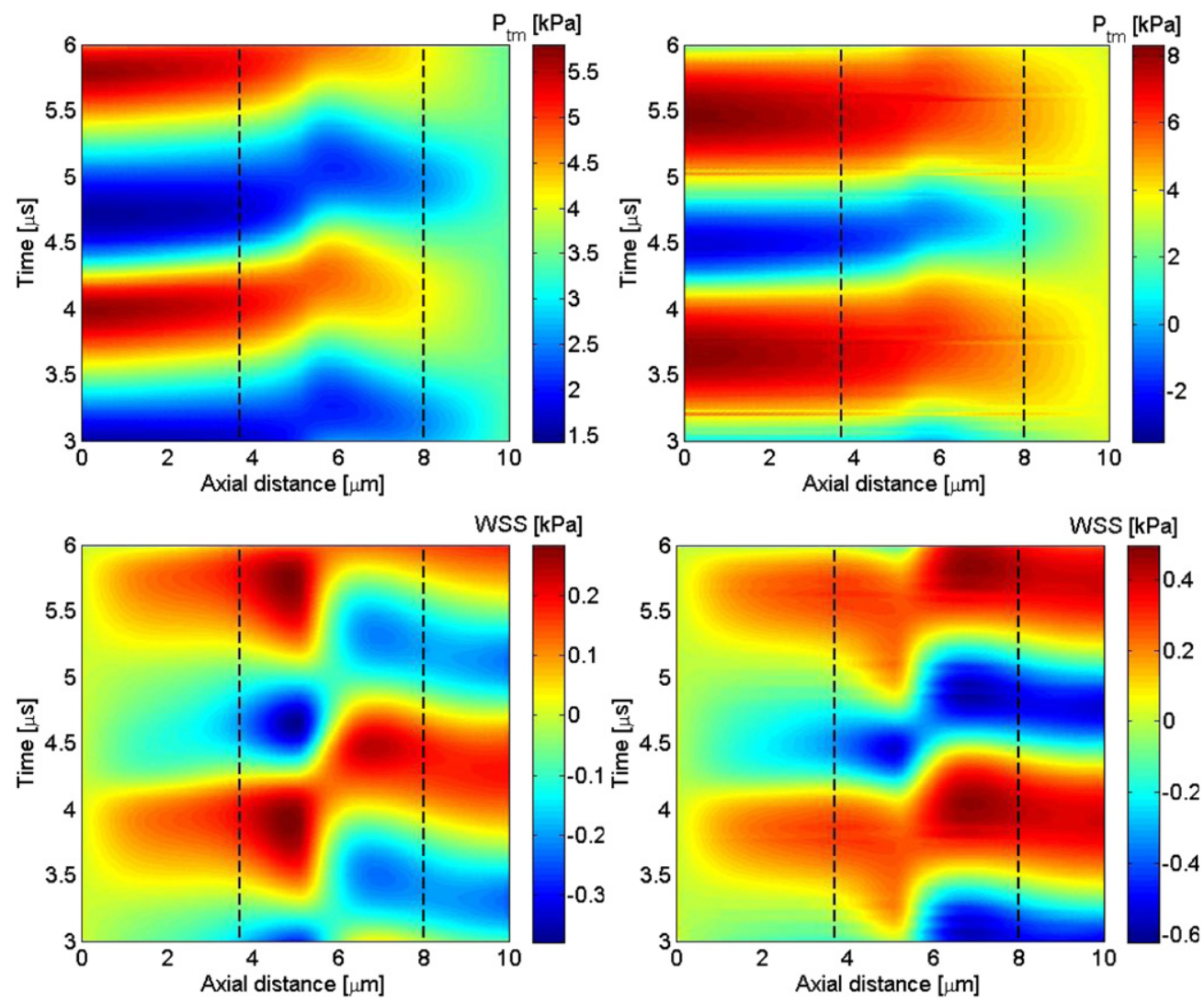

Figure 14. Comparison of $P_{\mathrm{tm}}$ (top) and WSS (bottom) patterns for vessels with different stiffness. With $E=4 \mathrm{MPa}$ (left column), the $P_{\mathrm{tm}}$ distribution and the distal WSS patterns are shifted towards later time points compared to a vessel with $E=9 \mathrm{MPa}$ (right column). In the more compliant vessel, the WSS regions appear to become more disjunct and the kink in the pressure distribution is more pronounced.

\subsection{Vessel dilation}

In all investigated setups, the maximum displacement of the vessel wall is of the order of $1-1.7 \%$ of the initial vessel radius. Cumulated oscillatory flows across the outlet reached up to $0.5 \%$ (case 1) and $1.3 \%$ (case 2) of the initial vessel volume. Mass imbalance induced by the numerical errors was low, remaining below $0.015 \%$ of the initial overall liquid mass throughout the entire simulation for all cases.

Maximum dilation values are observed in the proximal vessel region. As the dilation is directly related to the values of transmural pressure, this comportment is expected with view of the distribution of transmural pressure (figures 9 and 11). Taking into account $P_{\mathrm{tm}}$ and the acquired dilation, we computed the corresponding average distensibility values using equation (8). The results scale with Young's modulus of the vessel. The distensibilities amount to $1.05 \cdot 10^{-4} \mathrm{mmHg}^{-1}$ for $E=8 \mathrm{MPa}, 1.35 \cdot 10^{-4} \mathrm{mmHg}^{-1}$ for $E=6 \mathrm{MPa}$ and $2.1 \cdot 10^{-4} \mathrm{mmHg}^{-1}$ for $E=4 \mathrm{MPa}$. 


\section{Discussion}

Our investigations imply that RBCs play an important role in shaping the mechanical conditions induced by intravascular MB oscillations. RBCs impact the local distribution as well as spatial gradients of transmural pressure and WSS. We observe WSS values significantly above physiological levels and an increase of mechanical stress and pressure readings at the endothelium with increasing vessel rigidity.

\subsection{Transmural pressure and dilation}

The transmural pressure, i.e. the pressure difference between the lumen-endothelium interface and the perivascular space, creates forces on the vessel structure. It is an important characteristic quantity of the mechanical state of a vessel causing dilation and, consequently, the straining of the endothelial membrane. TJs bridging the intercellular clefts may be weakened, partially compromised or even completely disintegrated under such strains. This would cause the opening of paracellular pathways and ultimately enhance BBB permeability. After reestablishment of physiological conditions, a reformation of TJs may take place in less than 24 h (Gonzalez-Mariscal et al 1985).

Transmural pressure values that surpass a certain threshold will lead to straining beyond levels sustainable by the vessel structure, resulting in breach of vessel integrity. The examination of the temporal development of transmural pressure and its gradients may offer insight into the dynamic loading of the vessel during ultrasound-induced stable MB cavitation and aid in establishing safe margins for FUS-induced BBBD.

The $P_{\mathrm{tm}}$ distribution is monotonous in the $z$-direction with highest absolute values close to the MB. Deviations from that pattern are found in the vicinity of the RBC (figures 9 and 11). This causes a maximum absolute dilation of the vessel with accompanying peak strain values at the location of the MB. The kink in the pressure patterns leads to a highly localized peak in $\mathrm{g} P_{\mathrm{tm}}$ (figure 10), which causes high strain gradients in the vessel structure in the vicinity of the RBC.

Maximum transmural pressure values of up to $8.1 \mathrm{kPa}$ (case 1) and $17.8 \mathrm{kPa}$ (case 2) (60.7 and $133 \mathrm{mmHg}$, respectively) were found. Interestingly, these values do not depend strongly on whether and in which form RBCs are included in the simulations, and they are slightly higher for a configuration without RBC. This is due to partially impeded reflux of fluid from the inlet towards the bubble in the presence of the RBC. The channel between the RBC and the vessel wall acts as a contracting-expanding nozzle that imposes an adverse pressure gradient onto the reflux. To compensate for the insufficient reflux in the MB contraction phase, the vessel contracts more in the proximal region in the case of a rigid RBC. This leads to lower stress levels in the vessel structure and, consequently, to less pressure on the fluid. While this effect is also present in the configuration with a flexible and mobile $\mathrm{RBC}$, it can partly be compensated by shifting the RBC with the flow and thus increasing the pressure in the proximal region.

In contrast, the gradient values and patterns show a remarkable dependence on the chosen RBC model. They reach up to $2.43 \mathrm{kPa}_{\mu \mathrm{m}}^{-1}$ (case 1) and $9.8 \mathrm{kPa} \mu \mathrm{m}^{-1}$ (case 2). These gradients lead to strong local variations in dilation and, consequently, in strain along the endothelium. This imbalance in load along the cell membranes or across cell-cell junctions could play a role in BBBD.

We observe peak vessel dilation of $1-1.7 \%$ of the initial vessel diameter. This translates to average capillary distensibilities of $1.05 \cdot 10^{-4}$ to $2.1 \cdot 10^{-4} \mathrm{mmHg}^{-1}$ for the range of $E$ 
values addressed, which corresponds to mostly indistensible structures. Reported values of capillary distensibility are in the range of $7-19 \cdot 10^{-4} \mathrm{mmHg}^{-1}$ in the relevant pressure range (Baldwin and Gore 1989). Considering the simple linear elastic model employed in our study, this compares well to our reported values, but indicates that our model vessel is slightly too rigid.

\subsection{Wall shear stress}

The endothelium is exposed to longitudinal forces resulting from flow shearing at the vessel wall. Local variations in WSS result in a straining of the vessel and of the TJs that connect the endothelial cells. This strain, similar to the one induced by $P_{\mathrm{tm}}$, may cause partial or complete failure of the TJs and lead to an opening of the BBB. The observed spatial gradients in WSS (figure 10) are largest in the vicinity of the RBCs owing to the disturbance in WSS patterns introduced by the RBC (figures 9 and 11). These high shear gradients occur due to flow between the inner vessel wall and the RBC that acts like a moving piston. The nozzle shape of the gap causes deceleration of the fluid, adding to the WSS gradient. Visible effects of these processes are the reported patterns of flow splitting and converging flow.

The high gWSS values expose the endothelial cells and their TJs to rapidly varying parallel straining forces, stretching or compressing the interface in longitudinal direction. The occurrence of those forces in a confined region close to the erythrocytes underlines the importance of RBCs during MB oscillation for BBBD.

Peak WSS values reach up to $0.47 \mathrm{kPa}$ (case 1 ) and $2.0 \mathrm{kPa}$ (case 2), which is substantially above the physiological regime of 2-10 Pa (Reneman et al 2006). This is due to peak flow speeds that are roughly three orders of magnitude higher than those under physiological conditions.

Again, these values do not depend strongly on the presence of RBCs or their modelling, but their gradients are affected markedly by the model choice. The retrieved values are of the same order of magnitude as those reported based on bubble microstreaming models by $\mathrm{Wu}$ (2002) and Forbes et al (2008) of up to 10 and $17 \mathrm{kPa}$, respectively. The WSS values reported by Vos et al (2011) at 25-300 kPa are considerably higher, which can be explained by their setup in which the MB touches the wall.

The peak gWSS values are found next to the RBC's location, reaching up to $0.79 \mathrm{kPa} \mu \mathrm{m}^{-1}$ (case 1) and $2.8 \mathrm{kPa} \mu \mathrm{m}^{-1}$ (case 2). They are caused by the characteristics of the flow around the RBC, which is governed by the motion of the RBC driven by pressure gradients.

Whether the herein reported force fluctuations are likely to inflict damage to the endothelium needs to be assessed via experiments. There are indications that endothelial cells may be able to endure such elevated stresses for a limited amount of time: while, to our knowledge, no data on the structural integrity of the capillary endothelium exists, studies by Leverett et al (1972) on RBCs and Tillmann et al (1984) on RBCs and platelets show that these cells can sustain shear stress above $1 \mathrm{kPa}$ for an exposure time of more than $100 \mu \mathrm{s}$ and even higher values for shorter durations.

Finally, fluid shear stress at the endothelium is also known to influence vasoregulation, endothelial cell morphology and function, and even vessel remodelling (Mazzag et al 2003). However, endothelial cells are most likely not capable of sensing and responding to high frequency flows such as those caused by FUS-induced MB oscillation (Barakat 2001). 


\subsection{MB model validation}

The MB motion is prescribed by a modified RPE (3) (4) that takes into account the influence of confinement inside the vessel as well as the effects of an encapsulating MB shell. This approach limits the MB shape to a spherical form. As pointed out by Qin and Ferrara (2006), the dynamics of a MB in a vessel with diameter over ten times larger than the MB diameter are well described by the unconstrained RPE. The introduced extensions of the RPE allow for a further reduction of this limit.

In our simulations the local maximum variation in pressure across the MB surface reaches up to $1.1 \mathrm{kPa}$. This is small compared to the absolute intraluminal pressure, which is of the order of $100 \mathrm{kPa}$. Furthermore, the shell increases the MB stiffness considerably (Hoff et al 2000) and limits aspheric motion. Consequently, a significant deformation of the $\mathrm{MB}$ at the given moderate expansion ratios is not expected.

Finally, the central topic of the study at hand is the mechanical state at the vessel wall, which is defined by the far-field flow conditions rather than the state at the MB surface. Farfield conditions are determined predominantly by the overall volume displacement rate of the $\mathrm{MB}$ rather than by its shape, assuming that no MB fragmentation occurs. Fragmentation can lead to substantial forces at the endothelium through shock-wave generation and jet formation (Postema et al 2004), which is not considered in this work.

\subsection{RBC modelling}

The results show a significant influence of the presence of flexible, mobile RBCs on the flow patterns and, consequently, on the mechanical conditions at the vessel wall. While the presence of blood cells may not influence MB dynamics significantly (Stride and Saffari 2004), it shows a marked effect on the induced fluid dynamics, substantially disturbing the $P_{\mathrm{tm}}$ and WSS patterns compared to a configuration without RBCs (figure 9).

To our knowledge, this is the first time that the influence of RBCs is taken into account in the context of MB enhanced BBBD. RBCs are modelled as elastic, axially mobile parachute shaped solids. This treatment respects all degrees of freedom of the RBC under the restriction of an axisymmetric system. However, it does not consider friction forces that may arise due to contact between RBC and the endothelial surface layer (see section 4.5). This effect could reduce $\mathrm{RBC}$ displacement and with it the shear forces generated by the flow between RBC and vessel wall.

We modelled the RBCs as elastic solids with the same Young's modulus as that of the vessel. While clearly lower values of Young's modulus and more complex constitutive models have been reported for RBCs in their relaxed state, there is no published quantitative data on the comportment of RBCs in the here relevant dynamic state. We justify the matching of moduli between vessel and RBC as follows: radial deformation of the RBC is limited by the stiffness of the vessel, while global axial deformation will be limited by the translational degree of freedom. Local deformations that may also lead to surface waves on the RBCs would clearly be influenced by a lower modulus, but we do not expect these to have any significance on the mechanical state of the vessel.

\subsection{Endothelial surface layer}

The luminal endothelium surface is covered by a layer of membrane-bound macromolecules forming a gel-like structure of approximately $0.5 \mu \mathrm{m}$ thickness referred to as glycocalyx (Pries et al 2000). It is conceivable that the glycocalyx will have a marked effect on pressure and shear conditions acting on the endothelium of capillaries, especially in the area between RBC 
and vessel wall. The glycocalyx in contact with erythrocytes may possibly inhibit flow past the RBCs to a large extent. This would render the shear and pressure conditions uniquely dependent on the displacement and deformation of the RBC.

The rim of the RBC slides along the glycocalyx during motion but may not penetrate into this layer (Vink and Duling 1996). During MB-induced motion, RBCs are subjected to displacement velocities that are up to three orders of magnitude higher than those under physiological conditions. In addition, they experience backward motion. Under these conditions, RBCs may experience significantly higher resistance caused by the friction with the glycocalyx compared to physiological conditions, which may limit axial RBC mobility or increase shear forces on the EC layer due to a direct mechanical coupling to the RBC through the glycocalyx.

To our knowledge, the interaction of the glycocalyx with oscillating MBs in capillaries has not been investigated yet, and only limited data exists that would allow for accurate modelling of this system. We have neglected this interaction in the model at hand, but do note that the effect of the glycocalyx should be revisited upon availability of relevant quantitative experimental data.

\section{Conclusions}

A spatially selective opening of the BBB may be attained by means of MB enhanced FUS application. Assuming a predominantly mechanical BBBD pathway, two prominent actors in this process were identified: WSS and transmural pressure. Both parameters impose straining forces on the endothelial cells and their TJs. Partial or full disintegration of TJs is a purported pathway for BBBD.

At the length scales considered, blood can no longer be regarded as a homogeneous fluid, but should be seen as a solution containing various quasi-solid constituents. The appearance of remarkable features in the WSS and $P_{\text {tm }}$ distributions and specifically the highly localized peaks in their spatial gradients in the vicinity of those constituents, represented here by RBCs as their most abundant type, underlines the necessity of taking their presence into account.

Our numerical results indicate the occurrence of substantially increased pressure levels at the endothelium and shear stresses of several orders of magnitude above physiological values during MB oscillation. Further investigations will be necessary to assess whether these pressure and shear stress distributions could be responsible for BBBD.

A correlation of mechanical conditions derived from simulation with experimental assessment of BBBD under similar conditions could help identify critical physical features in the process and aid in the establishment of a parameter space within which safe BBBD can take place. The intention of our research was to develop a tool that can complement experimental studies to gain a more complete picture of the process of MB enhanced and FUS-induced BBBD.

\section{Acknowledgment}

We would like to express our gratitude for the funding of this research by the Swiss National Science Foundation through NCCR Co-Me.

\section{References}

Abbott N J, Patabendige A A K, Dolman D E M, Yusof S R and Begley D J 2010 Structure and function of the blood-brain barrier Neurobiol. Dis. 37 13-25 
Abbott N J and Romero I A 1996 Transporting therapeutics across the blood-brain barrier Mol. Med. Today 2 106-13

Alonso A, Reinz E, Jenne J W, Fatar M, Schmidt-Glenewinkel H, Hennerici M G and Meairs S 2010 Reorganization of gap junctions after focused ultrasound blood-brain barrier opening in the rat brain J. Cereb. Blood Flow Metab. 30 1394-402

Apfel R E and Holland C K 1991 Gauging the likelihood of cavitation from short-pulse, low-duty cycle diagnostic ultrasound Ultrasound Med. Biol. 17 179-85

Baldwin A L and Gore R W 1989 Simultaneous measurement of capillary distensibility and hydraulic conductance Microvasc. Res. 38 1-22

Barakat A I 2001 A model for shear stress-induced deformation of a flow sensor on the surface of vascular endothelial cells J. Theor. Biol. 210 221-36

Baseri B, Choi J J, Tung Y S and Konofagou E E 2010 Multi-modality safety assessment of blood-brain barrier opening using focused ultrasound and definity microbubbles: a short-term study Ultrasound Med. Biol. 36 1445-59

Bertossi M, Virgintino D, Maiorano E, Occhiogrosso M and Roncali L 1997 Ultrastructural and morphometric investigation of human brain capillaries in normal and peritumoral tissues Ultrastruct. Pathol. 21 41-9

Cash J R and Karp A H 1990 A variable order Runge-Kutta method for initial-value problems with rapidly varying right-hand sides ACM Trans. Math. Softw. 16 201-22

Caskey C F, Qin S, Dayton P A and Ferrara K W 2009 Microbubble tunneling in gel phantoms J. Acoust. Soc. Am. 125 EL183-9

Cassot F, Lauwers F, Fouard C, Prohaska S and Lauwers-Cances V 2006 A novel three-dimensional computerassisted method for a quantitative study of microvascular networks of the human cerebral cortex Microcirculation 13 1-18

Chin C T, Lancee C, Borsboom J, Mastik F, Frijlink M E, de Jong N, Versluis M and Lohse D 2003 Brandaris 128: a digital 25 million frames per second camera with 128 highly sensitive frames Rev. Sci. Instrum. 74 5026-34

Choi J J, Pernot M, Small S A and Konofagou E E 2007 Noninvasive, transcranial and localized opening of the blood-brain barrier using focused ultrasound in mice Ultrasound Med. Biol. 33 95-104

Church C C 1995 The effects of an elastic solid-surface layer on the radial pulsations of gas-bubbles J. Acoust. Soc. Am. 97 1510-21

Collis J, Manasseh R, Liovic P, Tho P, Ooi A, Petkovic-Duran K and Zhu Y G 2010 Cavitation microstreaming and stress fields created by microbubbles Ultrasonics $\mathbf{5 0} 273-9$

de Jong N, Frinking P J, Bouakaz A, Goorden M, Schourmans T, Jingping X and Mastik F 2000 Optical imaging of contrast agent microbubbles in an ultrasound field with a 100-MHz camera Ultrasound Med. Biol. 26 487-92

Degroote J, Bathe K J and Vierendeels J 2009 Performance of a new partitioned procedure versus a monolithic procedure in fluid-structure interaction Comput. Struct. 87 793-801

Dejana E 2004 Endothelial cell-cell junctions: happy together Nature Rev. Mol. Cell Biol. 5 261-70

Demirdzic I and Peric M 1988 Space conservation law in finite volume calculations of fluid-flow Int. J. Numer. Methods Fluids 8 1037-50

Dove A 2008 Breaching the barrier Nature Biotechnol. 26 1213-5

Dulinska I, Targosz M, Strojny W, Lekka M, Czuba P, Balwierz W and Szymonski M 2006 Stiffness of normal and pathological erythrocytes studied by means of atomic force microscopy J. Biochem. Biophys. Methods $661-11$

Eichna L W 1942 Capillary blood pressure in man-direct measurements in the digits during arterial hypertension induced by paredrinol sulfate J. Clin. Invest. $21731-4$

Faez T, Goertz D and De Jong N 2011 Characterization of Definity ${ }^{\mathrm{TM}}$ ultrasound contrast agent at frequency range of 5-15 MHz Ultrasound Med. Biol. 37 338-42

Farhat C and Geuzaine P 2004 Design and analysis of robust ALE time-integrators for the solution of unsteady flow problems on moving grids Comput. Methods Appl. Mech. Eng. 193 4073-95

Fenart L, Casanova A, Dehouck B, Duhem C, Slupek S, Cecchelli R and Betbeder D 1999 Evaluation of effect of charge and lipid coating on ability of 60-nm nanoparticles to cross an in vitro model of the blood-brain barrier J. Pharmacol. Exp. Ther. 291 1017-22

Ferziger J H and Peric M 1995 Computational Methods for Fluid Dynamics (Berlin: Springer)

Fischer M, Franzeck U K, Herrig I, Costanzo U, Wen S, Schiesser M, Hoffmann U and Bollinger A 1996 Flow velocity of single lymphatic capillaries in human skin Am. J. Physiol Heart Circ. Physiol. 270 H358-63

Forbes M M, Steinberg R L and O'Brien W D 2008 Examination of inertial cavitation of Optison in producing sonoporation of Chinese hamster ovary cells Ultrasound Med. Biol. 34 2009-18

Frinking P J A and de Jong N 1998 Acoustic modeling of shell-encapsulated gas bubbles Ultrasound Med. Biol. 24 523-33 
Geuzaine P, Grandmont C and Farhat C 2003 Design and analysis of ALE schemes with provable second-order time-accuracy for inviscid and viscous flow simulations J. Comput. Phys. 191 206-27

Godin M, Bryan A K, Burg T P, Babcock K and Manalis S R 2007 Measuring the mass, density, and size of particles and cells using a suspended microchannel resonator Appl. Phys. Lett. 91123121

Goertz D E, de Jong N and van der Steen A F W 2007 Attenuation and size distribution measurements of Definity ${ }^{\mathrm{TM}}$ and manipulated Definity ${ }^{\mathrm{TM}}$ populations Ultrasound Med. Biol. 33 1376-88

Gonzalez-Mariscal L, de Ramírez B C and Cereijido M 1985 Tight junction formation in cultured epithelial-cells (MDCK) J. Membr. Biol. 86 113-25

Greis C 2004 Technology overview: SonoVue (Bracco, Milan) Eur. Radiol. 14 P11-5

Grover W H, Bryan A K, Diez-Silva M, Suresh S, Higgins J M and Manalis S R 2011 Measuring single-cell density Proc. Natl Acad. Sci. USA 108 10992-6

Haidekker M A, Tsai A G, Brady T, Stevens H Y, Frangos J A, Theodorakis E and Intaglietta M 2002 A novel approach to blood plasma viscosity measurement using fluorescent molecular rotors Am. J. Physiol. Heart Circ. Physiol. 282 H1609-14

Hinghofer-Szalkay H and Greenleaf J E 1987 Continuous monitoring of blood-volume changes in humans J. Appl. Physiol. 63 1003-7

Hinghofer-Szalkay H and Moser M 1986 Fluid and protein shifts after postural changes in humans Am. J. Physiol. 250 H68-75

Hoff L, Sontum P C and Hovem J M 2000 Oscillations of polymeric microbubbles: effect of the encapsulating shell J. Acoust. Soc. Am. 107 2272-80

Hogan J, Smith P, Heath D and Harris P 1986 The thickness of the alveolar capillary wall in the human-lung at high and low altitude Br. J. Dis. Chest $\mathbf{8 0} 13-8$

Howles G P, Bing K F, Qi Y, Rosenzweig S J, Nightingale K R and Johnson G A 2010 Contrast-enhanced in vivo magnetic resonance microscopy of the mouse brain enabled by noninvasive opening of the blood-brain barrier with ultrasound Magn. Reson. Med. 64 995-1004

Hynynen K 2008 Ultrasound for drug and gene delivery to the brain Adv. Drug Deliv. Rev. 60 1209-17

Hynynen K, McDannold N, Sheikov N A, Jolesz F A and Vykhodtseva N 2005 Local and reversible blood-brain barrier disruption by noninvasive focused ultrasound at frequencies suitable for trans-skull sonications Neuroimage 24 12-20

Hynynen K, McDannold N, Vykhodtseva N and Jolesz F A 2001 Noninvasive MR imaging-guided focal opening of the blood-brain barrier in rabbits Radiology 220 640-6

Issa R I 1986 Solution of the implicitly discretized fluid-flow equations by operator-splitting J. Comput. Phys. 62 40-65

Jasak H, Jemcov A and Tuković Ž 2007 OpenFOAM: a C++ library for complex physics simulations Proc. Int. Workshop on Coupled Methods in Numerical Dynamics (Dubrovnik, Croatia) pp 47-66

Jasak H and Tuković Ž 2007 Automatic mesh motion for the unstructured finite volume method Trans. Famena 30 1-18

Jayaweera A R, Edwards N, Glasheen W P, Villanueva F S, Abbott R D and Kaul S 1994 In vivo myocardial kinetics of air-filled albumin microbubbles during myocardial contrast echocardiography. Comparison with radiolabeled red blood cells Circ. Res. 74 1157-65

Kaul S 2008 Myocardial contrast echocardiography: a 25-year retrospective Circulation 118 291-308

Klotz A R and Hynynen K 2010 Simulations of the Devin and Zudin modified Rayleigh-Plesset equations to model bubble dynamics in a tube Electron. J. Tech. Acoust. 11 1-15

Leverett L B, Hellums J D, Alfrey C P and Lynch E C 1972 Red blood-cell damage by shear-stress Biophys. J. 12 257-73

Liu H L et al 2010 Magnetic resonance monitoring of focused ultrasound/magnetic nanoparticle targeting delivery of therapeutic agents to the brain Proc. Natl Acad. Sci. USA 107 15205-10

Lockman P R, Mumper R J, Khan M A and Allen D D 2002 Nanoparticle technology for drug delivery across the blood-brain barrier Drug Dev. Ind. Pharm. 28 1-13

Lowe G D O 1987 Blood rheology in vitro and in vivo Baillieres Clin. Haematol. 1 597-636

Maneeratana K 2000 Development of the finite volume method for non-linear structural applications PhD Thesis Department of Mechanical Engineering, Imperial College London

Marmottant P, van der Meer S, Emmer M, Versluis M, de Jong N, Hilgenfeldt S and Lohse D 2005 A model for large amplitude oscillations of coated bubbles accounting for buckling and rupture J. Acoust. Soc. Am. 118 3499-505

Marquet F, Tung Y S, Teichert T, Ferrera V P and Konofagou E E 2011 Noninvasive, transient and selective bloodbrain barrier opening in non-human primates in vivo PLOS ONE 6 e22598

Martynov S, Stride E and Saffari N 2009 The natural frequencies of microbubble oscillation in elastic vessels J. Acoust. Soc. Am. $1262963-72$ 
Mathura K R, Vollebregt K C, Boer K, De Graaff J C, Ubbink D T and Ince C 2001 Comparison of OPS imaging and conventional capillary microscopy to study the human microcirculation J. Appl. Physiol. 91 74-8

Mazzag B M, Tamaresis J S and Barakat A I 2003 A model for shear stress sensing and transmission in vascular endothelial cells Biophys. J. 84 4087-101

McDannold N, Vykhodtseva N and Hynynen K 2006 Targeted disruption of the blood-brain barrier with focused ultrasound: association with cavitation activity Phys. Med. Biol. 51 793-807

McDannold N, Vykhodtseva N and Hynynen K 2008a Blood-brain barrier disruption induced by focused ultrasound and circulating preformed microbubbles appears to be characterized by the mechanical index Ultrasound Med. Biol. 34 834-40

McDannold N, Vykhodtseva N and Hynynen K 2008b Effects of acoustic parameters and ultrasound contrast agent dose on focused-ultrasound induced blood-brain barrier disruption Ultrasound Med. Biol. 34 930-7

McDannold N, Vykhodtseva N, Raymond S, Jolesz F A and Hynynen K 2005 MRI-guided targeted bloodbrain barrier disruption with focused ultrasound: histological findings in rabbits Ultrasound Med. Biol. 31 1527-37

McHedlishvili G I and Varazashvili M N 1980 High erythrocyte concentration in blood circulating in the brain Bull. Exp. Biol. Med. 90 1479-81

Meairs S and Alonso A 2007 Ultrasound, microbubbles and the blood-brain barrier Prog. Biophys. Mol. Biol. 93 354-62

Meier-Ruge W, Hunziker O, Schulz U, Tobler H J and Schweizer A 1980 Stereological changes in the capillary network and nerve-cells of the aging human-brain Mech. Ageing Dev. 14 233-43

Miao H, Gracewski S M and Dalecki D 2008 Ultrasonic excitation of a bubble inside a deformable tube: implications for ultrasonically induced hemorrhage J. Acoust. Soc. Am. 124 2374-84

Miller M W, Miller D L and Brayman A A 1996 A review of in vitro bioeffects of inertial ultrasonic cavitation from a mechanistic perspective Ultrasound Med. Biol. 22 1131-54

Murphy ME and Johnson P C 1975 Possible contribution of basement-membrane to structural rigidity of blood capillaries Microvasc. Res. $9242-5$

Noguchi H and Gompper G 2005 Shape transitions of fluid vesicles and red blood cells in capillary flows Proc. Natl Acad. Sci. USA 102 14159-64

Nyborg W L 2001 Biological effects of ultrasound: development of safety guidelines. Part II: general review Ultrasound Med. Biol. 27 301-33

Plesset M S and Prosperetti A 1977 Bubble dynamics and cavitation Annu. Rev. Fluid Mech. 9 145-85

Postema M, Van Wamel A, Lancee C T and De Jong N 2004 Ultrasound-induced encapsulated microbubble phenomena Ultrasound Med. Biol. 30 827-40

Pries A R, Secomb T W and Gaehtgens P 2000 The endothelial surface layer Pflugers Arch. $440653-66$

Prosperetti A 1991 The thermal-behavior of oscillating gas-bubbles J. Fluid Mech. 222 587-616

Qin S P, Caskey C F and Ferrara K W 2009 Ultrasound contrast microbubbles in imaging and therapy: physical principles and engineering Phys. Med. Biol. 54 R27-57

Qin S P and Ferrara K W 2006 Acoustic response of compliable microvessels containing ultrasound contrast agents Phys. Med. Biol. 51 5065-88

Qin S P and Ferrara K W 2007 The natural frequency of nonlinear oscillation of ultrasound contrast agents in microvessels Ultrasound Med. Biol. 33 1140-8

Rand PW, Austin W H, Lacombe E and Hunt HE 1964 Viscosity of normal human blood under normothermic + hypothermic conditions J. Appl. Physiol. 19 117-22

Reneman R S, Arts T and Hoeks A P G 2006 Wall shear stress-an important determinant of endothelial cell function and structure-in the arterial system in vivo J. Vasc. Res. 43 251-69

Sassaroli E and Hynynen K 2004 Forced linear oscillation's of microbubbles in blood capillaries J. Acoust. Soc. Am. 115 3235-43

Sheikov N, McDannold N, Vykhodtseva N, Jolesz F and Hynynen K 2004 Cellular mechanisms of the blood-brain barrier opening induced by ultrasound in presence of microbubbles Ultrasound Med. Biol. 30 979-89

Sontum P C 2008 Physicochemical characteristics of Sonazoid ${ }^{\mathrm{TM}}$, a new contrast agent for ultrasound imaging Ultrasound Med. Biol. 34 824-33

Sponheim N, Hoff L, Waaler A, Muan B, Morris H, Holm S, Myrum M, Dejong N and Skotland T 1993 Albunex ${ }^{\mathbb{R}}$ —a new ultrasound contrast agent IEEE Conf. Publ. pp 103-8

Stride E 2005 Contrast Media in Ultrasonography ed E Quaia (Berlin: Springer) pp 31-42

Stride E and Saffari N 2003 Microbubble ultrasound contrast agents: a review Proc. Inst. Mech. Eng. 217 429-47

Stride E and Saffari N 2004 Theoretical and experimental investigation of the behaviour of ultrasound contrast agent particles in whole blood Ultrasound Med. Biol. 30 1495-509 
Stucker M, Huntermann C, Bechara F G, Hoffmann K and Altmeyer P 2004 Capillary blood cell velocity in periulcerous regions of the lower leg measured by laser Doppler anemometry Skin Res. Technol. 10 174-7

Tillmann W, Reul H, Herold M, Bruss K H and Vangilse J 1984 In vitro wall shear measurements at aortic-valve prostheses J. Biomech. 17 263-79

Tu J, Guan J F, Qiu Y Y and Matula T J 2009 Estimating the shell parameters of SonoVue ${ }^{\mathbb{R}}$ microbubbles using light scattering J. Acoust. Soc. Am. 126 2954-62

Tu J, Hwang J H, Matula T J, Brayman A A and Crum L A 2006 Intravascular inertial cavitation activity detection and quantification in vivo with Optison Ultrasound Med. Biol. 32 1601-9

Tuković Ž 2005 Finite volume method on domains of varying shape PhD Thesis Faculty of Mechanical Engineering and Naval Architecture, University of Zagreb

Tuković Ž and Jasak H 2007 Updated Lagrangian finite volume solver for large deformation dynamic response of elastic body Trans. Famena 31 55-70

Tuković $\breve{Z}$ and Jasak H 2012 A moving mesh finite volume interface tracking method for surface tension dominated interfacial fluid flow Comput. Fluids $\mathbf{5 5} 70-84$

Tung Y S, Vlachos F, Choi J J, Deffieux T, Selert K and Konofagou E E 2010 In vivo transcranial cavitation threshold detection during ultrasound-induced blood-brain barrier opening in mice Phys. Med. Biol. 55 6141-55

Vink H and Duling B R 1996 Identification of distinct luminal domains for macromolecules, erythrocytes, and leukocytes within mammalian capillaries Circ. Res. 79 581-9

Vos H J, Dollet B, Versluis M and de Jong N 2011 Nonspherical shape oscillations of coated microbubbles in contact with a wall Ultrasound Med. Biol. 37 935-48

Vykhodtseva N, McDannold N and Hynynen K 2008 Progress and problems in the application of focused ultrasound for blood-brain barrier disruption Ultrasonics 48 279-96

Weller H G, Tabor G, Jasak H and Fureby C 1998 A tensorial approach to computational continuum mechanics using object-oriented techniques Comput. Phys. 12 620-31

Wu J R 2002 Theoretical study on shear stress generated by microstreaming surrounding contrast agents attached to living cells Ultrasound Med. Biol. 28 125-9

Zheng H R, Dayton P A, Caskey C, Zhao S K, Qin S P and Ferrara K W 2007 Ultrasound-driven microbubble oscillation and translation within small phantom vessels Ultrasound Med. Biol. 33 1978-87

Zieman S J, Melenovsky V and Kass D A 2005 Mechanisms, pathophysiology, and therapy of arterial stiffness Arterioscler. Thromb. Vasc. Biol. 25 932-43

Zudin Y B 1992 Analog of the Rayleigh equation for the problem of bubble dynamics in a tube J. Eng. Phys. Thermophys. $63672-5$

Zuk A, Targosz-Korecka M and Szymonski M 2011 Effect of selected drugs used in asthma treatment on morphology and elastic properties of red blood cells Int. J. Nanomed. 6 249-57 